TRANSACTIONS OF THE

AMERICAN MATHEMATICAL SOCIETY

Volume 361, Number 6, June 2009, Pages 3001-3030

S 0002-9947(08)04497-8

Article electronically published on December 23, 2008

\title{
CERTAIN OPTIMAL CORRESPONDENCES BETWEEN PLANE CURVES, II: EXISTENCE, LOCAL UNIQUENESS, REGULARITY, AND OTHER PROPERTIES
}

\author{
DAVID GROISSER
}

\begin{abstract}
This paper is a companion to the author's paper (this volume), in which several theorems were proven concerning the nature, as infinitedimensional manifolds, of the shape-space of plane curves and of spaces of certain curve-correspondences called bimorphisms. In Tagare, O'Shea, Groisser, 2002 , a class of objective functionals, depending on a choice of cost-function $\Gamma$, was introduced on the space of bimorphisms between two fixed curves $C_{1}$ and $C_{2}$, and it was proposed that one define a "best non-rigid match" between $C_{1}$ and $C_{2}$ by minimizing such a functional. In this paper we use the Nash Inverse Function Theorem to show that for strongly convex functions $\Gamma$, if $C_{1}$ and $C_{2}$ are $C^{\infty}$ curves whose shapes are not too dissimilar (specifically, are $C^{j}$ close for a certain finite $j$ ), and neither is a perfect circle, then the minimum of a certain regularized objective functional exists and is locally unique. We also study certain properties of the Euler-Lagrange equation for the objective functional, and obtain regularity results for "exact matches" (bimorphisms for which the objective functional achieves its absolute minimum value of 0 ) that satisfy a genericity condition.
\end{abstract}

\section{INTRODUCTION}

In TOG] a theory initially introduced in T] was developed for certain kinds of correspondences, termed bimorphisms, between simple closed regular plane curves of differentiability class at least $C^{2}$. This paper is a sequel to $[\mathrm{G}$, to which (along with [TOG] and [T] ) we refer the reader for the definition of bimorphisms and the motivation for studying them. To avoid repeating a large amount of material, this paper is not self-contained; the reader is advised to have a copy of [G] at hand.

In the present paper we prove an existence/uniqueness theorem (Theorem 1.1 below) for "optimal" bimorphisms determined by any objective-functional of a certain type. We also study some other properties of optimal bimorphisms, such as their regularity in certain cases, and make some observations on the associated Euler-Lagrange equation.

A bimorphism between two simple closed $C^{2}$ plane curves $C_{1}, C_{2}$ is a certain type of curve $\Sigma$ in the torus $C_{1} \times C_{2}$; for a precise definition see [G]. Given a "cost function" $\Gamma: \mathbf{R} \rightarrow \mathbf{R}$ - a continuous, even function achieving a minimum value of 0 uniquely at 0 , with $\Gamma(|x|)$ increasing in $|x|$ - one can define an objective functional

Received by the editors April 5, 2004 and, in revised form, February 11, 2007.

2000 Mathematics Subject Classification. Primary 53A04, 49K15.

Key words and phrases. Shape analysis, shape space, non-rigid correspondence, plane curve, bimorphism. 
on the set of bimorphisms by

$$
J_{\left(C_{1}, C_{2}\right)}(\Sigma)=\int_{\Sigma} \Gamma\left(\left|\frac{d \mu_{1}}{d s_{\Sigma}}\right| \kappa_{1}\left(\mu_{1}\left(s_{\Sigma}\right)\right)-\left|\frac{d \mu_{2}}{d s_{\Sigma}}\right| \kappa_{2}\left(\mu_{2}\left(s_{\Sigma}\right)\right)\right) d s_{\Sigma} .
$$

Here $s_{\Sigma}$ is an arclength parameter along $\Sigma$ (with respect to a Riemannian metric on $C_{1} \times C_{2}$ in which each factor has length 1$), s_{\Sigma} \mapsto\left(\mu_{1}\left(s_{\Sigma}\right), \mu_{2}\left(s_{\Sigma}\right)\right) \in C_{1} \times C_{2}$ is the corresponding parametrization of $\Sigma$, the vertical bars denote norm of a vector in $\mathbf{R}^{2}$, and $\kappa_{i}$ is the curvature of $C_{i}$. In $\mathrm{G}$ ] we observed that instead of viewing (1.1) as a functional on a certain space of curves in $C_{1} \times C_{2}$-hence a functional whose domain depends on $C_{1}$ and $C_{2}$-we can view 1.1. as defining a family of functionals $\Sigma^{\text {int }} \mapsto J\left(\Sigma^{\text {int }} ; \kappa_{1}, \kappa_{2}\right)$, parametrized by the curvature functions $\kappa_{1}, \kappa_{2}$, on a fixed space $\mathcal{B}^{\text {int } ~-~ a ~ c e r t a i n ~ s p a c e ~ o f ~ c u r v e s, ~ " i n t e r n a l ~ b i m o r p h i s m s ", ~ i n ~ t h e ~}$ standard torus $S^{1} \times S^{1}$.

It is not at all clear how one might attempt to prove existence or (local) uniqueness of a minimum of $J_{\left(C_{1}, C_{2}\right)}$ directly from the Euler-Lagrange equation (see \$4). However, the viewpoint above enables an Implicit-Function Theorem approach to the existence/uniqueness problem. If $C_{1}=C_{2}$ then $J_{\left(C_{1}, C_{2}\right)}$ achieves its absolute minimum of zero at the bimorphism corresponding to the identity map $C_{1} \rightarrow C_{2}$. In terms of the parametrized family of functionals on $\mathcal{B}^{\text {int }}$, this says that for all curvature functions $\kappa_{0}$, the functional $J\left(\cdot ; \kappa_{0}, \kappa_{0}\right)$ achieves its absolute minimum, namely zero, at the internal bimorphism $\Delta$ corresponding to the identity map $S^{1} \rightarrow S^{1}$. What one could hope to deduce from this using the Implicit Function Theoremwere it applicable here - is that for all curves sufficiently close to $C_{1}$ in a suitable topology, there exists a locally unique bimorphisms $\Sigma$ minimizing the corresponding functional $J\left(\cdot ; \kappa_{1}, \kappa_{2}\right)$.

As discussed in $\mathrm{G}$, the chief problem with this approach is that virtually none of the hypotheses of the standard (Banach-space) Implicit Function Theorem are satisfied, no matter what finite degree of diffentiability is imposed on the curves $C_{i}$. Nonetheless, by restricting to $C^{\infty}$ curves and using an implicit-function theorem derived from the Nash-Moser Inverse Function Theorem (see $[\mathrm{H}]$ ), we are able to prove an existence/uniqueness theorem for minima of a slightly perturbed (regularized) version of $J$. Our perturbation replaces $J$ by $J^{\varepsilon}:=J+\varepsilon Q$, where $\varepsilon>0$ and $Q\left(\Sigma^{\text {int }}\right)$ is the length of the curve $\Sigma^{\text {int }} \subset S^{1} \times S^{1}$. The functional $Q$ penalizes deviation from "uniformity", a perfectly uniform internal bimorphism $\Sigma^{\text {int }}$ being one in which, under a parametrization, the two factors of $S^{1} \times S^{1}$ are traversed at identically equal rates; for further discussion see $\$ 5$.

As in $\mathrm{G}$, by a based curve we will mean a pair $C_{b}=(C, q)$, where $C$ is a simple closed plane curve and $q \in C$. In $[\mathrm{G}, \S 1]$ we defined the space $\widetilde{\mathcal{S}}_{\infty}$ of shapes of $C^{\infty}$, based, simple closed curves in the plane, and the related space $\mathcal{S}_{\infty}$ of shapes of unbased curves. For any two based curves $C_{1, b}, C_{2, b}$ we defined the pseudo-identity $\left(C_{1}, C_{2}\right)$-bimorphism $\Sigma_{\mathrm{id}}\left(C_{1, b}, C_{2, b}\right) \subset C_{1} \times C_{2}$, and the space $\mathcal{B}_{\infty,+}\left(C_{1}, C_{2}\right)$ of $C^{\infty}$ orientation-preserving $\left(C_{1}, C_{2}\right)$-bimorphisms. The perturbed functional $J^{\varepsilon}$ determines a functional $J_{\left(C_{1, b}, C_{2, b}\right)}^{\varepsilon}: \mathcal{B}_{\infty,+}\left(C_{1}, C_{2}\right) \rightarrow \mathbf{R}$. Using this terminology we can state our main existence/uniqueness theorem:

Theorem 1.1. Let $\Gamma$ in (1.1) be $C^{2}$, assume that $\Gamma^{\prime \prime}(0)>0$, and fix $\varepsilon>0$. Let $C_{0, b}$ be a based, $C^{\infty}$, simple closed curve in $\mathbf{R}^{2}$ with based shape $\left[C_{0, b}\right] \in \widetilde{\mathcal{S}}_{\infty}$. Assume that $C_{0, b}$ is not a perfect circle. Then there exist $j_{1}, j_{2}<\infty$ and an open $C^{j_{1}}$ neighborhood $U$ of $\left[C_{0, b}\right]$ in $\widetilde{\mathcal{S}}_{\infty}$ such that for all based curves $C_{1, b}, C_{2, b}$ with $\left[C_{i, b}\right] \in$ 


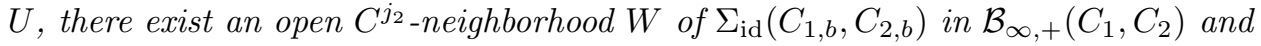
a unique $\left(C_{1}, C_{2}\right)$-bimorphism $\Sigma \in W$ at which the restriction of $J_{\left(C_{1, b}, C_{2, b}\right)}^{\varepsilon}$ to $W$ is minimized.

A useful corollary of Theorem 1.1 is the special case in which one of the based curves $C_{1, b}, C_{2, b}$, is $C_{0, b}$ itself. It may also be possible to use Theorem 1.1 to prove results for $C^{j}$ curves, $j<\infty$, by taking limits of minimizers for $C^{\infty}$ curves.

We actually prove a stronger version of this theorem, Theorem 5.4, whose statement requires terminology introduced later, and which gives a certain type of uniformity to the neighborhoods $W$ in Theorem 1.1] in essence these neighborhoods are determined purely by $C_{0, b}$.

Note that the local uniqueness of minimizers asserted in Theorem 1.1 is the best one can expect. For example, if one of the curves has a $2 \pi / n$ rotational symmetry for some $n>1$, minimizers will never be unique. Even absent any symmetry, there is no reason why two distant $\left(C_{1}, C_{2}\right)$-bimorphisms cannot both minimize $J_{\left(C_{1}, C_{2}\right)}$, although generically one would expect an absolute minimum to be achieved uniquely.

Theorem 1.1 implies a result for unbased curves, but the local uniqueness aspect is more awkward to state:

Corollary 1.2. Let $\Gamma$ and $\varepsilon$ be as in Theorem 1.1 and let $\pi: \widetilde{\mathcal{S}}_{\infty} \rightarrow \mathcal{S}_{\infty}$ be the natural projection. Let $C_{0}$ be a $C^{\infty}$ simple closed curve in $\mathbf{R}^{2}$, not a perfect circle, representing the unbased shape $\left[\left[C_{0}\right]\right] \in \mathcal{S}_{\infty}$. There exist $j_{1}, j_{2}<\infty$, and an open neighborhood $\hat{U}$ of $\left[\left[C_{0}\right]\right]$ in $\mathcal{S}_{\infty}$ of the form $\pi(U)$ for some open $C^{j_{1}}$-neighborhood $U$ in $\widetilde{\mathcal{S}}_{\infty}$ of a based representative $\left[C_{0, b}\right]$ of $\left[\left[C_{0}\right]\right]$, such that for all curves $C_{1}, C_{2}$ with $\left[\left[C_{i}\right]\right] \in \hat{U}$, there exist based representatives $C_{i, b}$ of $C_{i}$, a $C^{j_{2}}$ neighborhood $W$ of $\Sigma_{\mathrm{id}}\left(C_{1, b}, C_{2, b}\right)$ in $\mathcal{B}_{\infty,+}\left(C_{1}, C_{2}\right)$, and a unique $\left(C_{1}, C_{2}\right)$-bimorphism $\Sigma \in W$ minimizing $J_{\left(C_{1, b}, C_{2, b}\right)}^{\varepsilon} \mid W$.

A useful corollary of Theorem 1.1 is the special case in which one of the based curves $C_{1, b}, C_{2, b}$ is $C_{0, b}$ itself. It may also be possible to use Theorem 1.1 to prove results for $C^{j}$ curves, $j<\infty$, by taking limits of minimizers for $C^{\infty}$ curves, but we do not pursue that in this paper.

The reason for restricting to $C^{\infty}$ curves in Theorem 1.1-i.e. why one cannot set up the problem in such a way as to apply the Banach-space Implicit Function Theorem - is not obvious; we refer the reader to $G$. However, viewing the theorem as a means to understand whether one should expect the functional $J_{\left(C_{1, b}, C_{2, b}\right)}^{\varepsilon}$ to achieve a minimum in practical applications, the theorem's restriction to $C^{\infty}$ curves should not be a problem, since a digitized curve is no more a $C^{1}$ (or even $C^{0}$ ) object than it is a $C^{\infty}$ object, and one can do $C^{\infty}$ interpolation almost as easily as one can do piecewise linear interpolation. The requirement that the shapes of the curves be not too dissimilar - i.e. that their based curvature functions are close to that of $C_{0, b}$, hence to each other - should also not be an issue in practical applications. For example, if the two curves are epicardial outlines in successive echocardiographic pictures taken a short time apart, one would not expect drastic changes in the outline of the heart.

We also remark that while, in this paper, the regularization term $\varepsilon Q$ was added to $J$ purely to enable the proof of a theorem, in applications one almost always includes in the objective functional a term that penalizes deviation from uniformity, and $\varepsilon$ (or a similar parameter) need not be small (cf. [FB, T] ). However, there are 
good mathematical reasons why the unregularized functional $J$ is of interest, so it is reasonable to ask what happens as $\varepsilon \rightarrow 0$. This is another question not pursued here; we remark only that it may be possible to obtain from the methods of this paper an existence/uniqueness theorem for the unregularized functional, provided the curves $C_{1}, C_{2}$ are not convex (in addition to having shapes that are close). This approach is doomed to fail in the convex case, since for convex curves a minimum of $J_{\left(C_{1}, C_{2}\right)}$ always exists and is never unique (Theorem 2.5).

Because the Banach-space Implicit Function Theorem cannot be applied to the existence/uniqueness problem for minima of (1.1), in G] we prove that the space $\mathcal{M}_{\infty}^{\text {int }}$ - the space of $C^{\infty}$ curves homotopic to $C^{\infty}$ bimorphisms - is a tame Fréchet manifold (see the Appendix of [G] for definitions of "tame" and "Fréchet manifold"), in which the space $\mathcal{B}_{\infty}^{\text {int }}$ of $C^{\infty}$ bimorphisms is the closure of an open set. We also prove there that the based-shape space $\widetilde{\mathcal{S}}_{\infty}$ is a tame Fréchet manifold, although this fact is not strictly necessary for the proof of Theorem 1.1. This enables us to use the following "Tame Implicit Function Theorem" [G, Theorem 5.3] to prove Theorem 1.1 .

The remainder of this paper is organized as follows. For $2 \leq j \leq \infty$, formula (1.1) defines a "grand objective functional" $\tilde{J}=\tilde{J}^{\Gamma}: \widetilde{\mathcal{M}}_{j}=\widetilde{\mathcal{M}}_{j}^{\text {int }} \times \widetilde{\mathcal{K}}_{j-2} \times \widetilde{\mathcal{K}}_{j-2} \rightarrow \mathbf{R}$ by

$$
\tilde{J}\left(p ; \kappa_{1}, \kappa_{2}\right)=J_{\left(C_{1}, C_{2}\right)}(\Sigma),
$$

where $C_{i}$ is the normalized curve associated with the normalized curvature function $\kappa_{i}$ and where $\Sigma$ is the $\left(C_{1}, C_{2}\right)$-h-bimorphism associated to $\left(p, \kappa_{1}, \kappa_{2}\right)$ as in [G, $\left.\S 2\right]$. In $₫ 2$ we investigate the regularity of exact matches, the bimorphisms at which $\tilde{J}$ achieves its absolute minimum value 0 . In $₫ 3$ we derive the first variation formula for the functionals $\tilde{J}_{\left(C_{1}, C_{2}\right)}:=\tilde{J}\left(\cdot ; \kappa_{1}, \kappa_{2}\right)$, and the second variation at an exact match. We see there why a regularization term is needed for Theorem 1.1, 44 is devoted to a discussion of the Euler-Lagrange equation of the unregularized functional. We observe there that even though the functional $\tilde{J}_{\left(C_{1}, C_{2}\right)}$ is variationally awkward if we choose $\Gamma(x)=|x|$, as is often desired in practice (see, e.g., [FB), the EulerLagrange equation for $\tilde{J}_{\left(C_{1}, C_{2}\right)}$ for the choice $\Gamma(x)=|x|^{n}$ has a very nice limit as $n \downarrow 1$. In $\$ 5$ we add the regularization term to $\tilde{J}$ and state the main result of this paper, Theorem 5.4 of which Theorem 1.1 is a corollary. 86 is devoted to the (rather long and technical) proof of Theorem 5.4. For the background material concerning "tame calculus" on Fréchet spaces and Fréchet manifolds, we refer the reader to the Appendix of [G].

Notation and terminology in this paper are as in G. In particular, $S^{1}$ denotes the Riemannian manifold $\mathbf{R} / \mathbf{Z}$ with the metric inherited from $\mathbf{R}$ and with basepoint $0 ; S^{1} \times S^{1}$ is the corresponding flat torus; and for the Riemannian metrics on $\mathbf{R}^{2}$ and $S^{1} \times S^{1}$ we use both dot-product notation and parenthesis notation, according to convenience. Unless otherwise specified, "(parametrized) curve" means "simple, closed, oriented, (parametrized) regular curve" (either in the plane or a torus) of whatever differentiability class is specified. If no differentiability class is specified, $C^{2}$ is always assumed (except where explicitly stated otherwise), so that the curvature of a plane curve is always defined and continuous. For definitions of the spaces $\widetilde{\mathcal{K}}_{j}, \mathcal{K}_{j}, \widetilde{\mathcal{M}}_{j}^{\text {int }}, \mathcal{M}_{j}^{\text {int }}, \widetilde{\mathcal{M}}_{j}^{\text {int }, c}, \widetilde{\mathcal{B}}_{j}^{\text {int }}, \mathcal{B}_{j}^{\text {int }}$, and $\widetilde{\mathcal{B}}_{j}^{\text {int }, c}$ see [G, $\left.\S 2\right]$; for $\mathcal{M}_{j}$ and $\mathcal{B}_{j}$ see [G, §3]. 


\section{EXACT MATCHES}

In this section we consider certain $C^{l}$ bimorphisms between $C^{j}$ curves for which $1 \leq l<j$, observing that the formula (1.2) makes perfectly good sense for all $\left(p, \kappa_{1}, \kappa_{2}\right) \in \widetilde{\mathcal{M}}_{1}^{\text {int }} \times \widetilde{\mathcal{K}}_{0} \times \widetilde{\mathcal{K}}_{0}$. The fact that $\tilde{J}(\cdot) \geq 0$ motivates the following definition.

Definition 2.1. An exact $C^{l}$ match, $l \geq 1$, is a $C^{l}\left(C_{1}, C_{2}\right)$-bimorphism $\Sigma$ for some $C^{j}$ curves $C_{1}, C_{2}$, where $j \geq \max (l, 2)$, for which $J_{\left(C_{1}, C_{2}\right)}(\Sigma)=0$. An exact match is an exact $C^{l}$ match for some $l$.

From (1.2), $\Sigma$ is an exact match if and only if

$$
\dot{p}_{1}(t) \kappa_{1}\left(p_{1}(t)\right)-\dot{p}_{2}(t) \kappa_{2}\left(p_{2}(t)\right) \equiv 0
$$

for some (and hence every) triple $\left(p, \kappa_{1}, \kappa_{2}\right) \in \widetilde{\mathcal{B}}_{j}^{\text {int }} \times \widetilde{\mathcal{K}}_{l-2} \times \widetilde{\mathcal{K}}_{l-2}$ representing $\Sigma$. Note that the condition to be an exact match is independent of $\Gamma$.

Trivially, identity bimorphisms are exact matches; this will be important in the proof of Theorem 1.1. However, exact matches exist between certain curves not of the same shape. Such bimorphisms were termed "exact non-rigid matches" in [TOG], where the following was proved.

Theorem 2.2 (Theorem 3 of TOG]). Let $C_{1}, C_{2}$ be simple, closed, regular, $C^{j}$ plane curves $(j \geq 2)$ with curvature functions $\bar{\kappa}_{1}, \bar{\kappa}_{2}$ respectively. Assume that the functions $\bar{\kappa}_{i}$ have only finitely many zeroes, each of finite and integral order (and near which $\bar{\kappa}_{i}$ is continuously differentiable to that order), and at least one of which represents an inflection point (a point at which curvature changes sign). Then an exact match between $C_{1}$ and $C_{2}$ exists if and only if the curves have the same number of inflection points and have identical "angular deviation strings" (such a string being the list, modulo cyclic permutations, of the angles through which the normal vector rotates between consecutive inflection points). When this condition is satisfied, an exact match is a one-to-one correspondence between the curves, matching up the inflection points in a manner consistent with the angular deviation strings.

Note that when the conditions above for an exact match between $C_{1}$ and $C_{2}$ are met, there is a unique exact match unless one of the angular deviation strings possesses some cyclic symmetry, in which case there are still only finitely many exact matches.

In general, exact non-rigid matches between curves satisfying the hypotheses of Theorem 2.2 are only $C^{1}$. The existence of such matches, together with (for $l<j$ ) the approximability of $C^{l}$ bimorphisms by $C^{j}$ bimorphisms, shows that the function $\mathcal{S}_{j} \times \mathcal{S}_{j} \rightarrow \mathbf{R}$

$$
\left(\left[C_{1}\right],\left[C_{2}\right]\right) \mapsto \inf \left\{\bar{J}([\Sigma]) \mid \Sigma \in \mathcal{B}_{j}\left(C_{1}, C_{2}\right)\right\},
$$

while symmetric, non-negative, and obeying the triangle inequality, is only a "semidistance function" of shapes: for two distinct shapes $\left[C_{1}\right],\left[C_{2}\right]$, the infimal value of $J_{\left(C_{1}, C_{2}\right)}^{\Gamma}$ can still be zero. However, the fact that exact non-rigid matches of the type discussed above are only $C^{1}$ also shows that, for general $C^{j}$ curves $C_{1}, C_{2}$ with $j>1$, one should not be surprised if the functional $J_{\left(C_{1}, C_{2}\right)}^{\Gamma}: \mathcal{B}_{j}\left(C_{1}, C_{2}\right) \rightarrow \mathbf{R}$ fails to achieve a minimum (on this domain) - it definitely fails to achieve one if $C_{1}$ and $C_{2}$ have different shapes but satisfy the conditions in Theorem 2.2 
Since the plane curves we are dealing with are always assumed to be least $C^{2}$, it would seem natural to work with bimorphisms that are at least $C^{2}$ unless forced to do otherwise. But we will see during the proofs in this section that there are no natural conditions on $C^{j}$ curves, for finite $j$, guaranteeing that exact matches between $C^{j}$ curves obeying these conditions will be $C^{j}$. The least restrictive conditions guaranteeing generically optimal regularity of exact matches seem to be those in Definition 2.4 below.

Condition 2.3 (Generic Condition). We say that a normalized curvature function satisfies the generic condition if it has only finitely many zeroes, at each of which the curvature is $C^{1}$ and vanishes to order exactly 1 (and hence corresponds to an inflection point of the associated normalized plane curve). We say that a plane curve satisfies the generic condition if its normalized curvature function relative to some (hence any) basepoint does.

Definition 2.4. A generic exact match (GEM) between two $C^{j}$ curves $C_{1}, C_{2}$ $(j \geq 2)$ is an exact match $\Sigma$ for which one of the following is true:

(1) $\Sigma$ is an identity bimorphism;

(2) $\Sigma$ is a bimorphism between two curves of strictly positive curvature (necessarily convex curves, and therefore having no inflection points); or

(3) $\Sigma$ is a bimorphism between two non-convex $C^{j}$ curves satisfying the generic condition.

We will see below that for $j \geq 3$, a GEM between two $C^{j}$ curves is always of class $C^{j-2}$, and under a slightly stronger condition at the inflection points, such a GEM will be $C^{j-1}$. Other than for identity bimorphisms, it is rare for an exact match between curves that are not $C^{j+1}$ to be $C^{j}$.

Non-convexity of the curves in Theorem 2.2 is crucial for the local uniqueness of exact matches. Strong convexity (meaning strictly positive curvature) of the two curves is simultaneously the best situation for existence of an exact match and the worst situation for uniqueness. This follows from the ideas in the proof of Theorem 2.2. but since this result was not mentioned explicitly there, we state and prove it here:

Theorem 2.5. Let $C_{1}, C_{2}$ be simple, closed, regular, positively oriented $C^{j}$ plane curves $(j \geq 2)$ whose curvature functions are strictly positive. Then given any pair of points $q_{1} \in C_{1}, q_{2} \in C_{2}$, there exists a $C^{j-1}$ exact match between $C_{1}$ and $C_{2}$ matching $q_{1}$ with $q_{2}$. Any such match is the graph of a $C^{j-1}$ diffeomorphism between the curves, and if $j \geq 3$ then there is a unique such exact match.

Proof. It suffices to consider normalized curves with their canonical parametrizations. Letting $\kappa_{i}$ denote the curvature function of $C_{i}$, for $s \in[0,1]$ define $\omega_{i}(s)=$ $\int_{0}^{s} \kappa_{i}(u) d u, i=1,2$, where $\kappa_{i}$ is the normalized curvature function of the based curve $\left(C_{i}, q_{i}\right)$. Since $\omega_{i}(s)$ is just the angle through which the tangent (or normal) vector rotates counterclockwise as we move along $C_{i}$ from the basepoint to the point with parameter value $s$, we have $\omega_{i}(1)=2 \pi$. The functions $\omega_{i}$ are monotone (hence invertible) and are $C^{j-1}$; by the (baby) Inverse Function Theorem their inverses are also $C^{j-1}$. Define $p_{1}(t)=t, p_{2}(t)=\omega_{2}^{-1}\left(\omega_{1}(t)\right)$; the latter is a composition of $C^{j-1}$ diffeomorpisms, hence a $C^{j-1}$ diffeomorphism $[0,1] \rightarrow[0,1]$ that induces a 
$C^{j-1}$ diffeomorphism of $S^{1}$. We have $\dot{p}_{1}(t) \equiv 1$ and

$$
\dot{p}_{2}(t)=\frac{1}{\omega_{2}^{\prime}\left(p_{2}(t)\right)} \omega_{1}^{\prime}(t)=\frac{\kappa_{1}(t)}{\kappa_{2}\left(p_{2}(t)\right)}
$$

implying (2.1). Hence $\left(p_{1}, p_{2}\right)$ is an exact match with the stated properties.

Now let $\left(\tilde{p}_{1}, \tilde{p}_{2}\right)$ be an exact match pairing $q_{1}$ with $q_{2}$. Since the curvature functions are positive (2.1) implies that any exact match $\left(\tilde{p}_{1}, \tilde{p}_{2}\right)$ must have $d \tilde{p}_{1} / d t$, $d \tilde{p}_{2} / d t$ both strictly positive for all $t$ since by definition of "bimorphism" both cannot vanish simultaneously. Hence there exists a reparametrization with $\tilde{p}_{1}(t)=$ $t$. From (2.1) we then have (2.2), with $p_{i}$ replaced by $\tilde{p}_{i}$. The function $\tilde{p}_{2}$ is continuous, and since $\kappa_{1}$ and $\kappa_{2}$ are $C^{j-2}$ functions, the right-hand side of (2.2) is $C^{k}$ if $\tilde{p}_{2}$ is $C^{k}$ and $k \leq j-2$. But then $d p_{2} / d t$ is $C^{k}$, so $\tilde{p}_{2}$ is $C^{k+1}$. Hence by induction $\tilde{p}_{2}$ is $C^{j-1}$, and thus the parametrized bimorphism is $C^{j-1}$.

Let $F(t, y):=\kappa_{1}(t) / \kappa_{2}(y)$. If $j \geq 3$, then $F$ and $\partial F / \partial y$ are continuous, so the fundamental existence/uniqueness theorem for ODEs ensures that any solution to (2.2) with given initial conditions is unique. Hence the parametrized bimorphism $\left(\tilde{p}_{1}, \tilde{p}_{2}\right)$ coincides with the parametrized bimorphism $\left(p_{1}, p_{2}\right)$ defined in the first paragraph.

For GEMs of non-convex curves, we have the following.

Theorem 2.6. Every GEM between non-convex $C^{j}$ curves is $C^{j-2}$. If at all inflection points the curvature functions are $C^{j-1}$, then the $G E M$ is $C^{j-1}$.

Curvature functions of $C^{j}$ curves are automatically $C^{j-2}$, so the criterion in the second sentence demands only one extra degree of differentiability at the inflection points.

Proof of Theorem 2.6. We extend the ideas used in [TOG] to prove Theorem 2.2, Since Theorem 2.2 shows that GEMS are at least $C^{1}$, it suffices to assume $j \geq 3$.

Let $\left(\left(p_{1}, p_{2}\right), \kappa_{1}, \kappa_{2}\right)$ represent a GEM $\Sigma$; we have $p_{i} \in C^{j}$ and $\kappa_{i} \in C^{j-2}$. Write $k_{i}=\kappa_{i} \circ p_{i}$. As noted in [TOG], for a given $t_{0}, k_{1}\left(t_{0}\right)=0$ iff $k_{2}\left(t_{0}\right)=0$, and between successive zeroes, neither $\dot{p}_{i}$ can vanish since both by (2.1) would have to vanish simultaneously. Since the $\kappa_{i}$ vanish to order exactly 1 , neither $\dot{p}_{i}$ can vanish at a zero of $\kappa_{i}$, since again both $\dot{p}_{i}$ would have to vanish simultaneously. Hence both $\dot{p}_{i}$ are both strictly positive, and after a reparametrization and a change of basepoints (so that a given pair of matched inflection points are the new basepoints), we may assume that $p_{1}(t) \equiv t$, that $p_{1}(0)=0=p_{2}(0)$, and that $\kappa_{1}(0)=0=\kappa_{2}(0)$.

Let $I$ be the largest open neighborhood of 0 on which there are no other zeroes of the $k_{i}$, and let $I_{-}, I_{+}$be the components of $I-\{0\}$ to the left and right of 0 respectively. On each of $I_{+}, I_{-}$, equation (2.2) holds. The same argument as in Theorem 2.5 shows that $p_{2}$ is $C^{j-1}$ on $I-\{0\}$, so the only question is what happens at 0 .

Let $l=j-1$ if the hypothesis in the second sentence of the theorem is met; otherwise let $l=j-2$. Since the $\kappa_{i}$ vanish to order exactly 1 at 0 , we have

$$
\kappa_{i}(s)=2 a_{i} s+h_{i}(s)
$$

where $a_{i}:=\kappa_{i}^{\prime}(0) / 2 \neq 0$ and where $h_{i}$ is a $C^{l}$ function with $h_{i}(0)=h_{i}^{\prime}(0)=0$. On $I-\{0\},(2.1)$ implies that $\operatorname{sign}\left(k_{1}\right)=\operatorname{sign}\left(k_{2}\right)$, so $\operatorname{sign}\left(a_{1}\right)=\operatorname{sign}\left(a_{2}\right)$. For simplicity we assume $a_{i}>0$; the argument is essentially the same if $a_{i}<0$. 
On $I$, define $\omega_{i}(s)=\int_{0}^{s} \kappa_{i}(u) d u$; thus

$$
\omega_{i}(s)=a_{i} s^{2}\left(1+f_{i}(s)\right)
$$

where

$$
f_{i}(s)=s^{-2} \int_{0}^{s} h_{i}(u) d u=\int_{0}^{1} s^{-1} h_{i}(s w) d w .
$$

(Here and below our convention is that all functions that we write for $s \neq 0$ with formulas such as " $s^{-1} h(s w)$ " are extended by continuity to $s=0$.) Note that $1+f_{i}(s)>0$ on $I$ since $\omega_{i}(s)>0$ for $s \in p_{i}^{-1}(I-\{0\})$.

Define

$$
F(s)=a_{2}^{1 / 2} s\left(1+f_{2}(s)\right)^{1 / 2}:=a_{2}^{1 / 2} s G(s) .
$$

From (2.3), $f_{2}$ is $C^{l}$ on $I-\{0\}$, and hence so is $F$; furthermore $s f_{2}(s)$ is $C^{l}$ on $I$. Also, since $h_{2}$ is $C^{l}$ and vanishes to second order at $0, s^{-1} h_{2}(s)$ is $C^{l-1}$ on $I$. (This follows by writing $h_{2}$ its $l^{\text {th }}$-degree Taylor polynomial plus a remainder. The remainder term $R_{k}$ in the $k^{\text {th }}$-order Taylor expansion at 0 of a $C^{l}$ function $h$ has the property that for $0 \leq n \leq l, x^{-n} R_{l}(x)$ is $C^{l-n}$.) Hence $f_{2}$ is $C^{l-1}$ on $I$. It follows that $G$ is also $C^{l-1}$ on $I$ and $C^{l}$ on $I-\{0\}$.

We claim that $F$ is $C^{l}$ on $I$. From the preceding, we already know that $F$ is $C^{l-1}$ on $I$ and $C^{l}$ on $I-\{0\}$. Thus it suffices to show that $\lim _{s \rightarrow 0}(s G(s))^{(l)}$ exists, and since $(s G(s))^{(l)}=s G^{(l)}(s)+l G^{(l-1)}(s)$ and $G \in C^{l-1}(I)$, this reduces to showing that $\lim _{s \rightarrow 0} s G^{(l)}(s)$ exists. On $I-\{0\}$ we have

$$
G^{(l)}(s)=\frac{1}{2}\left(1+f_{2}\right)^{-1 / 2} f_{2}^{(l)}(s)+\left(1+f_{2}\right)^{-l-1 / 2} P_{l}\left(f_{2}(s), f_{2}^{\prime}(s), \ldots, f_{2}^{(l-1)}(s)\right)
$$

for some universal polynomial $P_{l}$. Since $f_{2} \in C^{l-1}(I)$ and $f_{2}(0)=0$, it follows that $\lim _{s \rightarrow 0} s G^{(l)}(s)=\lim _{s \rightarrow 0} \frac{1}{2} s f_{2}^{(l)}(s)=\frac{1}{2}\left(\left(s f_{2}\right)^{(l)}(s)-l f_{2}^{(l-1)}(s)\right)$, which exists since $s f_{2}(s) \in C^{l}(I)$ and $f_{2} \in C^{l-1}(I)$. Thus $F$ is $C^{l}$ on $I$. Since $F^{\prime}(0)=a_{2}^{1 / 2} \neq 0$, the (baby) Inverse Function Theorem implies that on some open neighborhood $\hat{I}$ of 0 , $F^{-1}$ exists and is $C^{l}$.

Let $\omega_{i}^{+}=\left.\omega_{i}\right|_{\hat{I}_{+} \cup\{0\}}, \omega_{i}^{-}=\left.\omega_{i}\right|_{\hat{I}_{-} \cup\{0\}}$. As shown in TOG], $\omega_{i}^{ \pm}$is monotone on $\hat{I}_{ \pm} \cup\{0\}$ and $p_{2}(t)=\left(\omega_{2}^{ \pm}\right)^{-1}\left(\omega_{1}(t)\right)$. By its definition, $F= \pm\left(\omega_{2}^{ \pm}\right)^{1 / 2}$ on $\hat{I}_{ \pm} \cup\{0\}$, and implying $\left(\omega_{2}^{ \pm}\right)^{-1}(v)=F^{-1}\left( \pm v^{1 / 2}\right)$. Hence on $\hat{I}_{ \pm} \cup\{0\}$ we have $p_{2}(t)=F^{-1}\left( \pm \omega_{1}(t)^{1 / 2}\right)$. The same argument that showed that $F$ is $C^{l}$ shows that $\left(\omega_{1}^{ \pm}\right)^{1 / 2}$ is $C^{l}$ on $\hat{I}_{ \pm} \cup\{0\}$. Thus $p_{2}$ is $C^{l}$ on each sub-interval $\hat{I}_{ \pm} \cup\{0\}$, so to show that $p_{2}$ is $C^{l}$ on $I$, it suffices to show that its left-hand derivatives equal its right-hand derivatives at 0 .

For this, let $g_{i}(s)=\kappa_{i}(s) / s$; then $g_{i}$ is $C^{l-1}$ on an open neighborhood of 0 and $g_{i}(0) \neq 0$. We can rewrite (2.2) as

$$
\dot{p}_{2}(t) \frac{p_{2}(t)}{t}=\frac{g_{1}(t)}{g_{2}\left(p_{2}(t)\right)}
$$

Let $\sum_{n=0}^{l-1} c_{n, i} s^{n}$ be the $(l-1)^{\text {st }}$-degree Taylor polynomial of $g_{i}(s)$ at 0 , and let $\sum_{n=0}^{l} b_{n}^{ \pm} s^{n}$ be the $l^{\text {th }}$-degree Taylor polynomial of $\left.f_{2}\right|_{I_{ \pm} \cup\{0\}}$ at 0 . From (2.4) we have

$$
\begin{array}{r}
\left(b_{1}^{ \pm}+2 b_{2}^{ \pm} t+\cdots+l b_{l}^{ \pm} t^{l-1}+o\left(t^{l-1}\right)\right)\left(b_{1}^{ \pm}+b_{2}^{ \pm} t+\cdots+b_{l}^{ \pm} t^{l-1}+o\left(t^{l-1}\right)\right) \\
=\frac{c_{0,1}+c_{1,1} t+\cdots+c_{l-1,1} t^{l-1}+o\left(t^{l-1}\right)}{c_{0,2}+c_{1,2} p_{2}(t)+\cdots+c_{l-1,2} p_{2}(t)^{l-1}+o\left(p_{2}(t)^{l-1}\right)} .
\end{array}
$$


From this we have $\left(b_{1}^{ \pm}\right)^{2}=c_{0,1} / c_{0,2}=a_{1} / a_{2}$, and since $\dot{p}_{2}(t)>0$ everywhere this implies that $b_{1}^{+}=b_{1}^{-}=b_{1}:=\left(a_{1} / a_{2}\right)^{1 / 2}$. In the formal power-series expansion of the right-hand side of (2.5), the terms of order $t^{m}(m \leq l-1)$ involve the coefficients $b_{l}^{ \pm}$only for $l \leq m$, whereas on the left-hand side the coefficient of $t^{m}$ is $(m+2) b_{1} b_{m+1}^{ \pm}+$(universal polynomial in $b_{1}, b_{2}^{ \pm}, \ldots, b_{m-1}^{ \pm}$, and the $\left.\left\{c_{n, i}\right\}\right)$. Hence the $b_{i}^{ \pm}$are determined by recursion relations that involve only universal polynomials and the coefficients $c_{n, i}$. Since $b_{1}^{+}=b_{1}^{-}$, it follows that $b_{m}^{+}=b_{m}^{-}$for all $m \leq l$. Hence at 0 the $m^{\text {th }}$ left-hand derivative of $p_{2}$ at 0 equals the $m^{\text {th }}$ right-hand derivative. Therefore $p_{2}$ is $C^{l}$ on $I$.

Remark 2.7. Essentially the same proof shows that if $\Sigma$ is an exact match of $C^{j}$ curves satisfying the conditions in Theorem 2.2. and at a given pair of matched inflection points $\left(q_{1}, q_{2}\right)$ both curvatures vanish to equal order $n<\infty$, then $\Sigma$ is $C^{j-n-1}$ at $\left(q_{1}, q_{2}\right)$.

The corollary below summarizes the regularity behavior of the three types of GEMs.

Corollary 2.8. Let $C_{1}, C_{2}$ be a pair $C^{j}$ curves admitting a GEM $\Sigma$. If $\Sigma$ is an identity bimorphism, then $\Sigma$ is $C^{j}$; if $C_{1}$ and $C_{2}$ are strongly convex, then $\Sigma$ is $C^{j-1}$; and if $C_{1}$ and $C_{2}$ are non-convex, then $\Sigma$ is $C^{j-2}$ or, if the extra hypothesis the second sentence of Theorem 2.6 is satisfied, $C^{j-1}$. In particular, all GEMs of $C^{\infty}$ curves are $C^{\infty}$.

We remark that (2.2) shows why a non-identity GEM between curves that are $C^{j}$ but not $C^{j+1}$ is rarely $C^{j}$ : the curvature functions are only $C^{j-2}$, and usually there would not exist any function $p_{2}$ for which the quotient of the two $C^{j-2}$ functions in (2.2) would be $C^{j-1}$, let alone a function that satisfies this differential equation.

\section{First AND SECOND VARIATION FORMUlas}

Let $j \geq 2$ (with $j=\infty$ allowed). For $\kappa_{1}, \kappa_{2} \in \widetilde{\mathcal{K}}_{j-2}$, (1.2) determines a functional

$$
\begin{aligned}
\tilde{J}_{\left(\kappa_{1}, \kappa_{2}\right)}: \widetilde{\mathcal{M}}_{j}^{\text {int }} & \rightarrow \mathbf{R}, \\
\tilde{J}_{\left(\kappa_{1}, \kappa_{2}\right)}(p) & =\tilde{J}\left(p ; \kappa_{1}, \kappa_{2}\right) ;
\end{aligned}
$$

note that $\tilde{J}_{\left(\kappa_{1}, \kappa_{2}\right)}: \widetilde{\mathcal{M}}_{j}^{\text {int }} \rightarrow \mathbf{R}$ descends to a functional $J_{\left(\kappa_{1}, \kappa_{2}\right)}: \mathcal{M}_{j}^{\text {int }} \rightarrow \mathbf{R}$. For $i=1,2$ let $C_{i, b}$ be a fixed based $C^{j}$ curve with normalized curvature function $\kappa_{i}$. In this section we consider the first variation of the functionals $\tilde{J}_{\left(\kappa_{1}, \kappa_{2}\right)}: \widetilde{\mathcal{M}}_{j}^{\text {int }} \rightarrow$ $\mathbf{R}, \tilde{J}_{\left(\kappa_{1}, \kappa_{2}\right)}^{c}:=\left.\tilde{J}_{\left(\kappa_{1}, \kappa_{2}\right)}\right|_{\widetilde{\mathcal{M}}_{j}^{\text {int }, c}}: \widetilde{\mathcal{M}}_{j}^{\mathrm{int}, c} \rightarrow \mathbf{R}$, and $J_{\left(\kappa_{1}, \kappa_{2}\right)}: \mathcal{M}_{\infty}^{\mathrm{int}} \rightarrow \mathbf{R}$, as well as the second variations of these functionals at GEMs. We discuss later in this section why, in order to prove an existence/uniqueness theorem for minima, we are eventually forced to pass to the quotient $\mathcal{M}_{\infty}^{\text {int }}$, rather than do all our work in the "nicer" spaces $\widetilde{\mathcal{M}}_{j}^{\text {int }, c}, j<\infty$. But already we can see why having to pass to a quotient forces $j=\infty$ upon us: for $j<\infty$, the phrase "first variation of $J_{\left(\kappa_{1}, \kappa_{2}\right)}$ on $\mathcal{M}_{j}^{\text {int }}$ " would be meaningless, since $\mathcal{M}_{j}^{\text {int }}$ has no differentiable structure (see $\mathrm{G}$, Theorem 2.8(e)] and its proof).

Given a parametrized internal h-bimorphism $p=\left(p_{1}, p_{2}\right)$, let us write

$$
\begin{aligned}
\phi(t)=\phi_{p}(t) & :=\dot{p}_{1}(t) \kappa_{1}\left(p_{1}(t)\right)-\dot{p}_{2}(t) \kappa_{2}\left(p_{2}(t)\right), \\
L(t)=L_{p}(t) & :=\sqrt{\left(\dot{p}_{1}(t)\right)^{2}+\left(\dot{p}_{2}(t)\right)^{2}} .
\end{aligned}
$$


We continue to use "dot" for $d / d t$; we use "prime" to denote derivatives of functions of other variables (e.g., " $\kappa_{1}^{\prime}\left(p_{1}(t)\right)$ "). We may rewrite (3.1) as

$$
\tilde{J}_{\left(\kappa_{1}, \kappa_{2}\right)}(p)=\int_{0}^{1} \Gamma\left(\frac{\phi_{p}(t)}{L_{p}(t)}\right) L_{p}(t) d t .
$$

Lemma 3.1. If $\Gamma$ is $C^{l}$, then $\tilde{J}_{\left(\kappa_{1}, \kappa_{2}\right)}$ is $C^{\min \{l, j-2\}}$; hence so is the restricted functional $\tilde{J}_{\left(\kappa_{1}, \kappa_{2}\right)}^{c}$. The functional $J_{\left(\kappa_{1}, \kappa_{2}\right)}: \mathcal{M}_{\infty}^{\text {int }} \rightarrow \mathbf{R}$ is $C^{l}$.

Proof. Define $F: C^{0}\left(S^{1}\right) \oplus C^{0}\left(S^{1}\right) \rightarrow C^{0}\left(S^{1}\right)$ by $F\left(h_{1}, h_{2}\right)(t)=\Gamma\left(h_{1}(t)\right) h_{2}(t)$. It is easy to check that $F$ is $C^{l}$. Since $\kappa_{1}, \kappa_{2}$ are $C^{j-2}$, it is also easy to check that the maps $\widetilde{\mathcal{M}}_{j}^{\text {int }} \rightarrow C^{0}\left(S^{1}\right)$ defined by $p \mapsto \phi_{p}$ and $p \mapsto L_{p}$ are $C^{j-2}$ and $C^{\infty}$ respectively. Finally, the integration map $C^{0}\left(S^{1}\right) \rightarrow \mathbf{R}$ is $C^{\infty}$, so by the Chain Rule $\tilde{J}_{\left(\kappa_{1}, \kappa_{2}\right)}$ is $C^{\min \{l, j-2\}}$.

In [G, Remark 4.2] we defined local $C^{\infty}$ sections of the bundle $\widetilde{\mathcal{M}}_{\infty}^{\text {int }, c} \rightarrow \mathcal{M}_{\infty}^{\text {int }}$; i.e. $C^{\infty}$ maps $s$ : (open set in $\left.\mathcal{M}_{\infty}^{\text {int }}\right) \rightarrow \widetilde{\mathcal{M}}_{\infty}^{\text {int,c }}$ with the property that $\pi_{\widetilde{\mathcal{M}}^{c} \rightarrow \mathcal{M}}{ }^{\circ} s=$ id. Thus for any such map $J_{\left(\kappa_{1}, \kappa_{2}\right)}=\tilde{J}_{\left(\kappa_{1}, \kappa_{2}\right)}^{c} \circ s$. Since $\tilde{J}_{\left(\kappa_{1}, \kappa_{2}\right)}^{c}: \widetilde{\mathcal{M}}_{\infty}^{\mathrm{int}, c} \rightarrow \mathbf{R}$ is $C^{l}$, it follows that so is $J_{\left(\kappa_{1}, \kappa_{2}\right)}$.

Hence as long as the curves $C_{i}$ are $C^{3}$ and $\Gamma$ is $C^{1}$, the function $\tilde{J}_{\left(\kappa_{1}, \kappa_{2}\right)}$ is $C^{1}$ and we can compute its derivative (first variation). However, to compute the second variation or to find an Euler-Lagrange equation for $\tilde{J}_{\left(\kappa_{1}, \kappa_{2}\right)}$, we need to assume that the curves $C_{i}$ are $C^{4}$ and that $\Gamma$ is $C^{2}$. These restrictions, as well as the strict inequality

$$
\Gamma^{\prime \prime}(0)>0,
$$

are henceforth assumed except where stated otherwise. We also define the function $A=A^{\Gamma}: \mathbf{R} \rightarrow \mathbf{R}$ by

$$
A(x)=x \Gamma^{\prime}(x)-\Gamma(x) .
$$

Note that $A^{\prime}(x)=x \Gamma^{\prime \prime}(x)$ and hence that

$$
\begin{gathered}
A(0)=A^{\prime}(0)=\Gamma(0)=\Gamma^{\prime}(0)=0, \quad \text { and } \\
A(x)>0 \quad \text { for } x \neq 0 .
\end{gathered}
$$

Let $X \in T_{p} \widetilde{\mathcal{M}}_{j}^{\text {int }} \cong C^{j}\left(S^{1}\right) \oplus C^{j}\left(S^{1}\right)$ be a variation vector field; we may write $X=$ $\left(X_{1}, X_{2}\right)$, where $X_{i} \in C^{j}\left(S^{1}\right)$. We will compute $\left.\left(D \tilde{J}_{\left(\kappa_{1}, \kappa_{2}\right)}\right)\right|_{p}(X)$, the directional derivative ( $=$ variational derivative) of $\tilde{J}_{\left(\kappa_{1}, \kappa_{2}\right)}$ in the direction $X$. Temporarily letting $\delta$ denote the variation of any function with respect to $X$ at $p$, we have

$$
\begin{aligned}
\delta \tilde{J}_{\left(\kappa_{1}, \kappa_{2}\right)} & =\int_{0}^{1}\left\{\Gamma^{\prime}\left(\frac{\phi}{L}\right)\left(\frac{\delta \phi}{L}-\phi \frac{\delta L}{L^{2}}\right) L+\Gamma\left(\frac{\phi}{L}\right) \delta L\right\} d t \\
& =\int_{0}^{1}\left\{\Gamma^{\prime}\left(\frac{\phi}{L}\right) \delta \phi-A\left(\frac{\phi}{L}\right) \delta L\right\} d t .
\end{aligned}
$$

Since $\delta p_{1}=X_{1}$ and $\delta p_{2}=X_{2}$, we have

$$
\begin{aligned}
\delta \phi & =\dot{X}_{1} \kappa_{1} \circ p_{1}+\dot{p}_{1} \kappa_{1}^{\prime} \circ p_{1} X_{1}-\left(\dot{X}_{2} \kappa_{2} \circ p_{2}+\dot{p}_{2} \kappa_{2}^{\prime} \circ p_{2} X_{2}\right) \\
& =\frac{d}{d t}\left(X_{1} \kappa_{1} \circ p_{1}-X_{2} \kappa_{2} \circ p_{2}\right), \\
\delta L & =\frac{\dot{p} \cdot \dot{X}}{L} .
\end{aligned}
$$


Writing

$$
k_{1}:=\kappa_{1} \circ p_{1}, k_{2}:=\kappa_{2} \circ p_{2},
$$

we obtain the first variation formula

$$
\left.\left(D \tilde{J}_{\left(\kappa_{1}, \kappa_{2}\right)}\right)\right|_{p}(X)=\int_{0}^{1}\left\{\Gamma^{\prime}\left(\frac{\phi}{L}\right)\left(k_{1} X_{1}-k_{2} X_{2}\right)^{\cdot}-A\left(\frac{\phi}{L}\right) \frac{\dot{p} \cdot \dot{X}}{L}\right\} d t .
$$

If we decompose $X$ into normal and tangential components, writing $X=f \mathbf{N}_{p}+$ $\lambda \mathbf{T}_{p}$ as in [G, §2], the tangential component must drop out of (3.6) because of the parametrization-independence of $\tilde{J}_{\left(\kappa_{1}, \kappa_{2}\right)}$. We can also see this explicitly: defining the slope-angle function $\theta=\theta_{p}: S^{1} \rightarrow \mathbf{R} /(2 \pi \mathbf{Z})$ by

$$
\dot{p}_{1}=|\dot{p}| \cos \theta_{p}, \quad \dot{p}_{2}=|\dot{p}| \sin \theta_{p},
$$

the integrand of (3.6) equals

$$
-\Gamma^{\prime}\left(\frac{\phi}{L}\right) \frac{d}{d t}\left[\left(k_{1} \sin \theta+k_{2} \cos \theta\right) f\right]+A\left(\frac{\phi}{L}\right) f \dot{\theta}+\frac{d}{d t}\left(\lambda \Gamma\left(\frac{\phi}{L}\right)\right),
$$

and the last term integrates to zero. If we set

$$
\begin{aligned}
\hat{\phi}:=\hat{\phi}_{\left(p, \kappa_{1}, \kappa_{2}\right)}:=\phi / L & =k_{1} \cos \theta-k_{2} \sin \theta, \\
k:=k_{\left(p, \kappa_{1}, \kappa_{2}\right)} & :=k_{1} \sin \theta+k_{2} \cos \theta,
\end{aligned}
$$

we can write (3.6) more compactly:

$$
\left.\left(D \tilde{J}_{\left(\kappa_{1}, \kappa_{2}\right)}\right)\right|_{p}\left(f \mathbf{N}_{p}+\lambda \mathbf{T}_{p}\right)=\int_{0}^{1}\left\{-\Gamma^{\prime}(\hat{\phi})(k f)^{\cdot}+A(\hat{\phi}) f \dot{\theta}\right\} d t .
$$

Thus, when we later restrict our variational calculations to the manifold $\widetilde{\mathcal{M}}_{j}^{\text {int,c }}$, where most variation vector fields $X$ have non-zero tangential components, only the normal components will enter the first variation of $\tilde{J}_{\left(\kappa_{1}, \kappa_{2}\right)}^{c}$.

Now assume that $p$ parametrizes a GEM - an absolute minimum and therefore a critical point of $\tilde{J}_{\left(\kappa_{1}, \kappa_{2}\right)}$. To compute the second variation at $p$, we hold $X$ fixed and compute the derivative of (3.6) at $p$ in the direction $Y=\left(Y_{1}, Y_{2}\right)$. Since $\phi \equiv 0$ for a GEM, (3.3) implies that

$$
\begin{aligned}
\left.\left(H \tilde{J}_{\left(\kappa_{1}, \kappa_{2}\right)}\right)\right|_{p}(X, Y) & :=\left.\left(D^{2} \tilde{J}_{\left(\kappa_{1}, \kappa_{2}\right)}\right)\right|_{p}(X, Y) \\
& =\int_{0}^{1}\left\{\Gamma^{\prime \prime}(0) \frac{\delta \phi}{L}\left(k_{1} X_{1}-k_{2} X_{2}\right)^{\cdot}\right\} d t
\end{aligned}
$$

where now $\delta$ means variation in the direction $Y$. From (3.5), we then have

$$
\left.\left(H \tilde{J}_{\left(\kappa_{1}, \kappa_{2}\right)}\right)\right|_{p}(X, Y)=\Gamma^{\prime \prime}(0) \int_{0}^{1} \frac{1}{L}\left(k_{1} X_{1}-k_{2} X_{2}\right)^{\cdot}\left(k_{1} Y_{1}-k_{2} Y_{2}\right)^{\cdot} d t .
$$

Since $p$ parametrizes a GEM, the zeroes of $k_{1}$ and $k_{2}$ coincide, and $\operatorname{sign}\left(k_{1}\right)=$ $\operatorname{sign}\left(k_{2}\right)$ at every point where the curvature is nonzero. Writing $\operatorname{sign}(k)$ for this common sign, extended arbitrarily to the zeroes of the $k_{i}$, it follows from $k_{1} \cos \theta-$ $k_{2} \sin \theta=\phi \equiv 0$ that $k=\operatorname{sign}(k) \sqrt{k_{1}^{2}+k_{2}^{2}}$. If we write $X=f_{1} \mathbf{N}_{p}+\lambda_{1} \mathbf{T}_{p}, Y=$ $f_{2} \mathbf{N}_{p}+\lambda_{2} \mathbf{T}_{p}$, (3.10) simplifies to

$$
\left.\left(H \tilde{J}_{\left(\kappa_{1}, \kappa_{2}\right)}\right)\right|_{p}(X, Y)=\Gamma^{\prime \prime}(0) \int_{0}^{1} \frac{1}{L}\left(k f_{1}\right)^{\cdot}\left(k f_{2}\right)^{\cdot} d t .
$$

This Hessian is manifestly positive-semidefinite, as it had to be since exact matches are absolute minima, but for our implicit-function theorem approach to 
Theorem 1.1 we will need a Hessian that is strictly positive-definite. However, $\left.\left(H \tilde{J}_{\left(\kappa_{1}, \kappa_{2}\right)}\right)\right|_{p}$ has an infinite-dimensional nullspace that contains the space of all vector fields tangent to the image of $p$.

We can eliminate most of this degeneracy by restricting $\tilde{J}_{\left(\kappa_{1}, \kappa_{2}\right)}$ to $\widetilde{\mathcal{M}}_{j}^{\text {int,c }}$. The formula for $\left.\left(H \tilde{J}_{\left(\kappa_{1}, \kappa_{2}\right)}^{c}\right)\right|_{p}(X, Y)$ is of course still given by (3.11), but now $p$ is constrained to have constant speed, and $X$ and $Y$ are constrained to lie in $T_{p} \widetilde{\mathcal{M}}_{j}^{\text {int }, c} \cong T_{p}^{(2)} \mathcal{M}_{\infty}^{\text {int }} \oplus \mathbf{R} \mathbf{T}_{p}$ (see ([G] equations (2.21) and (2.23)); also $L$ is now a constant $\ell_{p}$, the length of the curve $\Sigma^{\text {int }}$ parametrized by $p$, and can be pulled outside the integral. However $N_{p}^{c}$, the nullspace of $\left.\left(H \tilde{J}_{\left(\kappa_{1}, \kappa_{2}\right)}^{c}\right)\right|_{p}$, still contains a 1-dimensional subspace, the space $\mathbf{R T}_{p}$ consisting of all constant multiples of the unit tangent vector field.

This 1-dimensional degeneracy was inherent from the start, since $\tilde{J}_{\left(\kappa_{1}, \kappa_{2}\right)}^{c}$ is invariant under the action of $S^{1}$ on $\widetilde{\mathcal{M}}_{j}^{\text {int,c }}$. To eliminate this degeneracy, the natural thing to do is to pass to the quotient $\widetilde{\mathcal{M}}_{j}^{\text {int,c }} / S^{1}=\mathcal{M}_{j}^{\text {int }}$. However, for finite $j$, $\mathcal{M}_{j}^{\text {int }}$ has no differentiable structure and it makes no sense to speak of a differentiable function on it - a major problem since we want to apply an implicit function theorem.

Unfortunately, this is not an instance in which one can circumvent singularities in a quotient $E / G$ by treating an object defined on $E / G$ as a $G$-equivariant object defined on $E$. The set of points in $\widetilde{\mathcal{M}}_{j}^{\text {int,c }}(j<\infty)$ at which the $S^{1}$-action fails to be differentiable is open and dense (and therefore so is the corresponding set of singular points in $\left.\mathcal{M}_{j}^{\text {int }}\right)$. I.e., the most serious problem already exists at the level of $\widetilde{\mathcal{M}}_{j}^{\text {int,c}}$; it is not caused by passing to the quotient $\mathcal{M}_{j}^{\text {int }}$. It is remarkable that what appears to be a very simple group action - rotations of the domain circle of (h-)bimorphisms - causes this very real problem, one that cannot be avoided by tricks such as completing the space of $C^{j}$ functions in a Sobolev norm or any other norm that involves only finitely many derivatives. Thus, to use an implicitfunction-theorem approach, we are forced to restrict attention to $\widetilde{\mathcal{M}}_{\infty}^{\text {int,c }}$ and its quotient $\mathcal{M}_{\infty}^{\text {int }}$.

The nullspace $N_{\Sigma}$ of $H J_{\left(\kappa_{1}, \kappa_{2}\right)}$ at $\Sigma$, the image of $p$ in $\mathcal{M}_{\infty}^{\text {int }}$, is the quotient space $N_{p}^{c} /\left(\mathbf{R T}_{p}\right)$ and is isomorphic to $N_{p}^{c} \cap \tilde{T}_{p}^{(2)} \mathcal{M}_{\infty}^{\text {int }}$, hence to $\left\{f \in C^{\infty}\left(S^{1}\right) \mid(k f)^{\cdot} \equiv\right.$ $0\}=\left\{f \in C^{\infty}\left(S^{1}\right) \mid k f \equiv\right.$ constant $\}$. We now see why the non-convex and strongly convex cases are different. If $C_{1}$ and $C_{2}$ are strongly convex, then $k>0$ everywhere, and the function $f=1 / k$ generates a 1-dimensional nullspace $N_{\Sigma}$. However, in the non-convex case, $k=0$ at some point, and hence the only possible constant value of $k f$ is 0 . But since $k$ vanishes only at isolated points, this implies $f \equiv 0$, and hence $N_{\Sigma}=\{0\}$.

Thus, at a GEM between non-convex curves, the Hessian $H=H J_{\left(C_{1}, C_{2}\right)}$ is strictly positive-definite. For infinite-dimensional manifolds, even of Banach type, strict positivity of the Hessian is not enough to deduce stability (local minimality); one needs strong positivity: $H(X, X) \geq c_{1}\|X\|^{2}$ for some $c_{1}>0$. In the Fréchet setting one needs strong positivity for some sufficiently strong norm; in our case it will turn out that the Sobolev norm of order 1 suffices. However, the Hessian at a GEM $\Sigma$ of non-convex curves is not strongly positive with respect to this norm, and we will see explicitly in $\$ 5$ that the derivative of the $L^{2}$ gradient of $J_{\left(C_{1}, C_{2}\right)}$ is not an isomorphism from $T_{\Sigma} \mathcal{M}_{\infty}^{\text {int }}$ to itself. (For a GEM it can be shown that 
the Hessian is strongly positive with respect to the $L^{2}$ norm, but this norm is not strong enough to ensure stability.) To deal with this problem, in $\$ 5$ we add to $J_{\left(C_{1}, C_{2}\right)}$ the regularizing term $\varepsilon Q$ mentioned in the Introduction.

\section{Minimality and the Euler-Lagrange equation}

In this section we fix a pair of based $C^{j}$ plane curves $C_{1, b}, C_{2, b}$ with normalized curvature functions $\kappa_{1}, \kappa_{2}$. We assume that $\Gamma$ is $C^{2}$ (except where otherwise stated) and $j \geq 3$, so that $\tilde{J}_{\left(\kappa_{1}, \kappa_{2}\right)}^{c}$ and $J_{\left(\kappa_{1}, \kappa_{2}\right)}$ are $C^{1}$ (Lemma 3.1), and we assume that $\Gamma$ is strongly convex $\left(\Gamma^{\prime \prime}(0)>0\right.$ for all $\left.x\right)$.

Integrating by parts the first term in the integral in (3.9), we have

$$
\left.\left(D \tilde{J}_{\left(\kappa_{1}, \kappa_{2}\right)}\right)\right|_{p}\left(f \mathbf{N}_{p}+\lambda \mathbf{T}_{p}\right)=\langle\tilde{Z}, f\rangle_{L^{2}},
$$

where

$$
\tilde{Z}:=\tilde{Z}_{\kappa_{1}, \kappa_{2}}(p):=k\left(\Gamma^{\prime}(\hat{\phi})\right)^{\cdot}+A(\hat{\phi}) \dot{\theta}
$$

(see (3.7 and (3.8)). Since we are computing on $\widetilde{\mathcal{M}}_{j}^{\text {int,c }}$ we have $L_{p} \equiv \ell_{p}$. Let us write

$$
k_{1}^{\prime}:=\kappa_{1}^{\prime} \circ p_{1}, k_{2}^{\prime}:=\kappa_{2}^{\prime} \circ p_{2} .
$$

Then $(\phi / L)^{\cdot}=\dot{\phi} / \ell_{p}=-k \dot{\theta}+\ell_{p}\left(k_{1}^{\prime} \cos ^{2} \theta-k_{2}^{\prime} \sin ^{2} \theta\right)$, so

$$
\tilde{Z}=\left(-k^{2} \Gamma^{\prime \prime}(\hat{\phi})+A(\hat{\phi})\right) \dot{\theta}+k \ell_{p} \Gamma^{\prime \prime}(\hat{\phi})\left(k_{1}^{\prime} \cos ^{2} \theta-k_{2}^{\prime} \sin ^{2} \theta\right) .
$$

We are interested in minimizing the objective functional only on the space $\widetilde{\mathcal{B}}_{j}^{\text {int }}$ of internal bimorphisms, not on the larger space $\widetilde{\mathcal{M}}_{j}^{\text {int }}$ of internal h-bimorphisms. However, for no $j$ is $\widetilde{\mathcal{B}}_{j}^{\text {int }}$ a manifold; it is a closed subset of $\widetilde{\mathcal{M}}_{j}^{\text {int }}$ with boundary

$$
\partial \widetilde{\mathcal{B}}_{j}^{\text {int }}=\left\{\left(p_{1}, p_{2}\right) \in \widetilde{\mathcal{M}}_{j}^{\text {int }} \mid \dot{p}_{i}(t) \geq 0 \forall t \text {, and } \exists t \text { for which } \dot{p}_{1}(t)=0 \text { or } \dot{p}_{2}(t)=0\right\} .
$$

The interior of $\widetilde{\mathcal{B}}_{j}^{\text {int }}$ - the set of points $p \in \widetilde{\mathcal{M}}_{j}^{\text {int }}$ for which $\dot{p}_{1}, \dot{p}_{2}$ are everywhere strictly positive inherits from $\widetilde{\mathcal{M}}_{j}^{\text {int }}$ the structure of a manifold modeled on $C^{j}\left(S^{1}, \mathbf{R}^{2}\right)$. All parametrized bimorphisms $p$ in the interior of $\mathcal{B}_{j}$ yield one-to-one correspondences between the first and second factors of $S^{1} \times S^{1}$ (in fact $p_{2} \circ p_{1}^{-1}$ is a diffeomorphism); all bimorphisms containing a segment-to-point correspondence lie in $\partial \widetilde{\mathcal{B}}_{j}^{\text {int }}$. Similar statements hold with $\widetilde{\mathcal{B}}_{j}^{\text {int }}, \widetilde{\mathcal{M}}_{j}^{\text {int }}$ replaced by $\widetilde{\mathcal{B}}_{j}^{\text {int }, c}, \widetilde{\mathcal{M}}_{j}^{\text {int }, c}$ respectively, or by $\mathcal{B}_{j}^{\text {int }}, \mathcal{M}_{j}^{\text {int }}$.

We now restrict our attention to constant-speed internal bimorphisms and the functional $\tilde{J}_{\left(\kappa_{1}, \kappa_{2}\right)}^{c}$. Because $\widetilde{\mathcal{B}}_{j}^{\text {int,c }}$ is not open in $\widetilde{\mathcal{M}}_{j}^{\text {int }, c}$, the condition that $\left.\tilde{J}_{\left(\kappa_{1}, \kappa_{2}\right)}^{c}\right|_{\mathcal{B}_{j}^{\text {int }, c}}$ have a relative minimum at $p$ is in general not simply $\tilde{Z}=0$. Since we are interested only with variations which remain within $\widetilde{\mathcal{B}}_{j}^{\text {int,c }}$, the function $f$ in (4.1) is constrained by

$$
\left\{\begin{aligned}
f(t) \geq 0 & \text { if } \theta(t)=0 \\
f(t) \leq 0 & \text { if } \theta(t)=\pi / 2
\end{aligned}\right.
$$

(Of course, this constraint is vacuous if $p$ is in the interior of $\widetilde{\mathcal{B}}_{j}^{\mathrm{int}, c}$.)

Assume that $\left.\tilde{J}_{\left(\kappa_{1}, \kappa_{2}\right)}^{c}\right|_{\tilde{\mathcal{B}}_{j}^{\text {int }, c}}$ has a local minimum at $p$; then the right-hand side of (4.1) must be non-negative for all variations within $\widetilde{\mathcal{B}}_{j}^{\text {int }, c}$. Suppose there exists $t_{0} \in S^{1}$ with $\theta\left(t_{0}\right)=0$ and $\tilde{Z}\left(t_{0}\right)<0$. From (4.3) $\tilde{Z}$ is continuous in $t$, so there exists 
an interval $I \subset S^{1}$, containing $t_{0}$, on which $\tilde{Z}$ is negative and on which $\theta$ is never $\pi / 2$. Let $f$ be a continuous non-negative function supported in $I$ with $f\left(t_{0}\right)>0$. Then $\int_{0}^{1} \tilde{Z} f d t<0$, so from (4.1) there exists a variation of $p$ within $\widetilde{\mathcal{B}}_{j}^{\text {int }, c}$ which decreases $\tilde{J}_{\left(\kappa_{1}, \kappa_{2}\right)}^{c}$. Thus we must have $\tilde{Z}\left(t_{0}\right) \geq 0$. Similarly, if $\theta\left(t_{0}\right)=\pi / 2$, then $\tilde{Z}\left(t_{0}\right) \leq 0$.

Let $R=R(p) \subset S^{1}$ be the closure of the set $\left\{t \in S^{1} \mid 0<\theta(t)<\pi / 2\right\}$, let $I_{0}=I_{0}(p)$ be the interior of the set $\left\{t \in S^{1} \mid \theta(t)=0\right\}$, and let $I_{\pi / 2}=I_{\pi / 2}(p)$ be the interior of the set $\left\{t \in S^{1} \mid \theta(t)=\pi / 2\right\}$. Then $S^{1}$ is the disjoint union of these three subsets, and the condition that $\left.\tilde{J}_{\left(\kappa_{1}, \kappa_{2}\right)}^{c}\right|_{\widetilde{\mathcal{B}}_{j}^{\text {int }, c}}$ has a local minimum at $p$ is: $\tilde{Z}(t)=0$ if $t \in R ; \tilde{Z}(t) \geq 0$ if $t \in I_{0}$; and $\tilde{Z}(t) \leq 0$ if $t \in I_{\pi / 2}$. The formula (4.3) simplifies considerably on $I_{0}$ and $I_{1}$, allowing us to rewrite the minimality criterion as

$$
\begin{cases}\tilde{Z} \equiv 0 & \text { on } R, \\ k_{2} k_{1}^{\prime} \geq 0 & \text { on } I_{0}, \text { with equality at } \partial I_{0}, \\ k_{1} k_{2}^{\prime} \geq 0 & \text { on } I_{\pi / 2}, \text { with equality at } \partial I_{\pi / 2} .\end{cases}
$$

(The equalities at the boundaries of $I_{0}$ and $I_{1}$ come from the continuity of $\tilde{Z}$.) Note that $S^{1}=R$ iff $\Sigma$ is a one-to-one, correspondence; if $\Sigma$ is not one-to-one then it contains a point-to-segment correspondence, and hence either $I_{0}$ or $I_{\pi / 2}$ is nonempty. Thus, a necessary condition for minimality is the Euler-Lagrange equation

$$
\tilde{Z} \equiv 0
$$

iff $\Sigma$ is one-to-one. This one-to-one criterion is a less restrictive condition than requiring $\Sigma$ to lie in the interior of $\widetilde{\mathcal{B}}_{j}^{\text {int }, c}$, but it excludes any bimorphism that matches a point of one curve with an arc of the other.

Defining $B(x)=B^{\Gamma}(x)=A^{\Gamma}(x) / \Gamma^{\prime \prime}(x)$, we can rewrite (4.6) as

$$
\dot{\theta}=\ell_{p} \frac{k\left(\cos ^{2} \theta k_{1}^{\prime}-\sin ^{2} \theta k_{2}^{\prime}\right)}{k^{2}-B(\hat{\phi})}:=\ell_{p} G\left(p_{1}, p_{2}, \theta\right),
$$

provided the denominator in (4.7) is nonzero. The Euler-Lagrange equation together with the definition of $\theta$ can then be written as the first-order system of ODEs

$$
\left\{\begin{aligned}
\dot{\theta} & =\ell_{p} G\left(p_{1}, p_{2}, \theta\right) \\
\dot{p}_{1} & =\ell_{p} \cos \theta \\
\dot{p}_{2} & =\ell_{p} \sin \theta
\end{aligned}\right.
$$

again assuming $\operatorname{denom}(G) \neq 0$.

Observe that the entire dependence of this system on $\Gamma$ is through the term involving $B$ in (4.7). To get more of a feel for this term, note that from (3.4) we have $B(0)=0$ and $B(x)>0$ for $x \neq 0$. In fact, independent of $\Gamma$, the Taylor expansion of $B$ at 0 is $\frac{1}{2} x^{2}+o\left(x^{2}\right)$ in general, and $\frac{1}{2} x^{2}+O\left(x^{4}\right)$ if $\Gamma$ is $C^{4}$. Thus one would expect the qualitative behavior of solutions of (4.8) for which the pointwisemismatch function $|\hat{\phi}|$ is uniformly small not to be very sensitive to the choice of $\Gamma$.

An interesting phenomenon occurs for $\Gamma$ of the form $x \mapsto|x|^{n}$, where $n$ need not be an integer. The only value of $n$ for which $\Gamma$ is $C^{2}$ everywhere and $\Gamma^{\prime \prime}(0)>0$ is 2. However, suppose we temporarily relax our regularity requirement and require 
only that $\Gamma$ be $C^{2}$ everywhere except at the origin, but still assume that $\Gamma^{\prime \prime}(x)>0$ for $x \neq 0$ - conditions satisfied by $|x|^{n}$ for all $n>1$. The first variation formula (3.9) still makes sense, and implies that for all $f$ that vanish wherever $\hat{\phi}$ does, (4.1) holds. This implies that if $\tilde{J}_{\left(\kappa_{1}, \kappa_{2}\right)}^{c}$ is minimized at $p$ then $\tilde{Z} \equiv 0$ on the set $U \subset S^{1}$ on which $\hat{\phi} \neq 0$; thus, dividing through by $\Gamma^{\prime \prime}(\hat{\phi})$, from (4.3) we have $\left(-k^{2}+B(\hat{\phi})\right) \dot{\theta}+k \ell_{p}\left(k_{1}^{\prime} \cos ^{2} \theta-k_{2}^{\prime} \sin ^{2} \theta\right) \equiv 0$ on $U$. Thus if the function $B$ extends continuously at 0 , then by continuity this equation holds throughout $S^{1}$, and (4.7) holds wherever the denominator is nonzero. For $\Gamma(x)=|x|^{n}$ with $n>1$, this is exactly what happens; we have $B(x)=\frac{1}{n} x^{n}$. For such $\Gamma$ the Euler-Lagrange equation is therefore

$$
\left(-k^{2}+\frac{1}{n} \hat{\phi}^{2}\right) \dot{\theta}+k \ell_{p}\left(k_{1}^{\prime} \cos ^{2} \theta-k_{2}^{\prime} \sin ^{2} \theta\right) \equiv 0
$$

(which we may write in the form (4.7) wherever $k^{2} \neq \frac{1}{n} \hat{\phi}^{2}$ ).

What is even more curious is that (4.9) makes perfectly good sense even if $n=1$, even though both $\Gamma^{\prime \prime}(x)$ and $A(x)$ are identically zero for $x \neq 0$. Thus while the variational problem with $\Gamma(x)=|x|$ is difficult to deal with directly, it may be possible to obtain solutions of this problem as solutions of the limiting EulerLagrange equation as $n \downarrow 1$, simply setting $n=1$ in (4.9). The $n=1$ case of (4.9) is esthetically the most pleasing of all, since the condition that the denominator in (4.8) be nonzero can be rewritten simply as

$$
k_{1} \sin (\theta-\pi / 4)+k_{2} \cos (\theta-\pi / 4) \neq 0 \neq k_{1} \cos (\theta-\pi / 4)-k_{2} \sin (\theta-\pi / 4) .
$$

Returning to the case in which $\Gamma$ is $C^{2}$ and strongly convex (or, more generally, $C^{2}$ everywhere except at the origin, with $\Gamma^{\prime \prime}(x)>0 \forall x \neq 0$ and with $\lim _{x \rightarrow 0} B(x)$ existing), note that a solution of (4.8) involves the specification of four objects: three real-valued functions $p_{1}, p_{2}, \theta$ and a positive number $\ell_{p}$. For such a quadruple to correspond to a constant-speed internal bimorphism, several conditions must be satisfied: $\ell_{p}$ must lie in $[\sqrt{2}, 2)$; the functions must be defined on the whole real line; $\theta$ must be periodic with period 1 and must take its values in $[0, \pi / 2]$; and $\int_{0}^{1} \dot{p}_{1}(t) d t=1=\int_{0}^{1} \dot{p}_{2}(t)$ (equivalently $\int_{0}^{1} \cos \theta(t) d t=\ell_{p}^{-1}=\int_{0}^{1} \sin \theta(t) d t$ ). From an ODE point of view, it is not at all obvious whether solutions to (4.8), subject to these constraints, exist. That is one reason we use an implicit-function theorem approach in this paper, rather than trying to prove existence and uniqueness directly from (4.8).

As noted above, the Euler-Lagrange equation (4.6) is a necessary condition for minimality only for one-to-one bimorphisms. One can construct a highly nongeneric example of a minimal bimorphism that contains a point-to-segment match as follows. Let $C_{1}$ be a curve that has a flat segment containing a point $p$, and let $C_{2}$ be identical except that the flat segment $S$ is longer. There is an exact match between $C_{1}$ and $C_{2}$ that matches $p$ to a flat sub-segment of $S$. However, this example is not very interesting in that a parametrization of it still obeys the Euler-Lagrange equation.

It is not clear whether minimal bimorphisms exist that do not obey (4.6), i.e. for which at least one of the extra two lines in (4.5) is relevant. However, if such bimorphisms do exist, then from (4.5) we can make certain observations about them. Before stating these, observe that the set $R(p)$ in (4.5) determines a parametrizationindependent set $R(\Sigma)$ in the bimorphism $\Sigma$ parametrized by $p$. 
Proposition 4.1. Let $j \geq 3$ and let $\Sigma$ be a bimorphism between $C^{j}$ plane curves $C_{1}, C_{2}$, that matches a point $q \in C_{2}$ with an arc $S \subset C_{1}$ and that minimizes the functional $J_{\left(C_{1}, C_{2}\right)}$ defined in (1.1). Let $\bar{\kappa}_{i}: C_{i} \rightarrow \mathbf{R}$ be the curvature function of $C_{i}, i=1,2$. Then either

(1) $\bar{\kappa}_{2}(q)=0$, or

(2) $\bar{\kappa}_{2}(q) \neq 0$ and $\bar{\kappa}_{1}$ is monotonic on $S$ (nondecreasing if $\bar{\kappa}_{2}(q)>0$, nonincreasing if $\left.\bar{\kappa}_{2}(q)<0\right)$.

In case (1), if $\bar{\kappa}_{1}$ and its derivative are nonvanishing at the endpoints of $S$, and if the zeroes in $R(\Sigma)$ of the derivative of $\theta_{\Sigma}$ do not accumulate at either endpoint of $S$, then the interior of $S$ contains either a vertex (a relative extremum of $\bar{\kappa}_{1}$ ) or an inflection point.

Proof. Let $p \in \widetilde{\mathcal{M}}_{j}^{\text {int }}$ be an internal bimorphism for which $\left(p, \kappa_{1}, \kappa_{2}\right)$ represents $\Sigma$, let $I_{0}^{0}=\left(t_{-}, t_{+}\right)$be the interior of $p_{1}^{-1}(S) \subset S^{1}$, let $\kappa_{i}$ be the normalized curvature functions corresponding to $\bar{\kappa}_{i}$, and let $k_{i}=\kappa_{i} \circ p_{i}$. Since $p_{2}$ (and therefore $k_{2}$ ) is constant on any connected component of $I_{0}(p)$, the breakdown into cases is immediate from the second line of (4.5); the only non-obvious assertion is the last sentence of the proposition.

Assume now that $k_{1}\left(t_{ \pm}\right) \neq 0 \neq k_{1}^{\prime}\left(t_{ \pm}\right)$(this is equivalent to the nonvanishing of $\bar{\kappa}_{1}$ and its derivative at endpoints of $S$ since on $I_{0}^{0}$ we have $\dot{p}_{1} \neq 0$ ). By our hypothesis on the zeroes of $\dot{\theta}$ in $R(\Sigma)$, for sufficiently small $\epsilon>0$ the function $\dot{\theta}$ does not vanish on $\left(t_{+}, t_{+}+\epsilon\right)$, and since $\theta \equiv 0$ in $I(p)$ the only possible sign for $\dot{\theta}$ on $\left(t_{+}, t_{+}+\epsilon\right)$ is positive. We therefore have $\theta>0$ throughout this interval as well, and therefore $\tilde{Z} \equiv 0$ on $\left(t_{+}, t_{+}+\epsilon\right)$.

On $I_{0}^{0}$ we have $k \equiv 0$ and $\hat{\phi} \equiv k_{1}$; thus as $\epsilon \rightarrow 0$ the denominator of (4.7), evaluated at $t_{+}$, tends to $-B\left(k_{1}\left(t_{+}\right)\right)<0$ (by the properties of $B$ noted earlier). Therefore if we take $\epsilon$ small enough, (4.7) will be satisfied on $\left[t_{+}, t_{+}+\epsilon\right)$, and the denominator in (4.7) will be negative throughout this interval.

In the numerator, the second factor tends to $k_{1}^{\prime}\left(t_{+}\right) \neq 0$, so we analyze the sign of the first factor $k=k_{1} \sin \theta+k_{2} \cos \theta$; the sign is not obvious since both terms vanish at $t_{+}$. However, we have $\dot{k}_{2}(t)=\kappa_{2}^{\prime}\left(p_{2}(t)\right) \dot{p}_{2}(t)=k_{2}^{\prime}(t) \ell_{p} \sin \theta(t)$, and since $\dot{\theta}>0$ on $\left(t_{+}, t_{+}+\epsilon\right)$, it follows that for $t$ in this interval,

$$
\left|k_{2}(t)\right|=\left|k_{2}(t)-k_{2}\left(t_{+}\right)\right| \leq\left(t-t_{+}\right) \sup \left\{\left|\dot{k}_{2}(s)\right| \mid s \in\left[t_{+}, t\right]\right\} \leq C\left(t-t_{+}\right) \sin \theta(t) .
$$

Thus for $t$ sufficiently close to $t_{+}$, if we take $\epsilon$ sufficiently small, $\left|k_{2} \cos \theta\right|(t)<$ $\left|k_{1} \sin \theta\right|(t)$ strictly, and the sign of $k(t)$ is that of $k_{1}(t)$. Thus the sign of the righthand side of (4.7) near $t_{+}$is $-\operatorname{sign}\left(k_{1}\left(t_{+}\right) k_{1}^{\prime}\left(t_{+}\right)\right)$, and since the left-hand side is positive, we must have $k_{1}\left(t_{+}\right) k_{1}^{\prime}\left(t_{+}\right)<0$.

A similar analysis shows that $k_{1}\left(t_{-}\right) k_{1}^{\prime}\left(t_{-}\right)>0$, so at least one of $k_{1}$ and $k_{1}^{\prime}$ must change sign in $S$, and the conclusion follows.

\section{Regularizing $\tilde{J}_{\left(C_{1}, C_{2}\right)}$}

Henceforth we assume that $\Gamma$ is $C^{\infty}$ and work only with (h-)bimorphisms and internal (h-)bimorphisms that are $C^{\infty}$; therefore we omit the subscript " $\infty$ " from various spaces (writing simply $\mathcal{M}^{\text {int }}$ for $\mathcal{M}_{\infty}^{\text {int }}$, etc.).

If we perform a variation on $\widetilde{\mathcal{M}}^{\text {int,c }}$ at $p$ in the direction $f \mathbf{N}_{p}+\lambda \mathbf{T}_{p}$, where $\lambda=\hat{\lambda}_{p}(f, a)$ for some $a \in \mathbf{R}$, then (using the notation (3.7)-(3.8)) we have the 
useful identities

$$
\begin{aligned}
\left.\delta \theta\right|_{p} & =\ell_{p}^{-1}\left(\dot{f}+\lambda \dot{\theta}_{p}\right), \\
\left.\delta \hat{\phi}\right|_{p} & =\ell_{p}^{-1}\left(\hat{\phi} \dot{\theta}_{p} f-(k f)+(\hat{\phi}) \dot{\lambda}\right), \\
\left.\delta k\right|_{p} & =\ell_{p}^{-1}\left\{\left(\hat{\phi}+k \dot{\theta}_{p}+\ell_{p}\left(k_{2}^{\prime}-k_{1}^{\prime}\right)\right) f+\hat{\phi} \dot{f}+\dot{k} \lambda\right\}, \quad \text { and } \\
\delta \ell_{p} & =-\left\langle f \dot{\theta}_{p}\right\rangle,
\end{aligned}
$$

as the reader may check. Henceforth we will often omit the subscript $p$.

A uniform map between plane curves $C_{1}$ and $C_{2}$ is a constant-speed map. In practical applications it is often desirable to add a term to $J_{\left(C_{1}, C_{2}\right)}$ that penalizes deviation from uniformity; cf. [T]. Under the factorization

$$
\mu=e_{\left(\kappa_{1}, \kappa_{2}\right)} \circ p
$$

discussed in [G, §2], a parametrized bimorphism $\mu$ is uniform if and only if the slope-angle function $\theta_{p}$ is constant. Because of the homotopy-class restriction in the definition of $\widetilde{\mathcal{M}}^{\text {int }}$, the only possible constant value of $\theta$ is $\pi / 4$. Thus a simple measure of deviation from uniformity is the parametrization-invariant functional $\tilde{Q}_{\text {temp }}: \widetilde{\mathcal{M}}^{\text {int }} \rightarrow \mathbf{R}$ given by

$$
\tilde{Q}_{\mathrm{temp}}(p)=\int_{0}^{1}\left(1-\cos \left(\theta_{p}(t)-\pi / 4\right)\right)|\dot{p}(t)| d t .
$$

However, observe that for any constant $\theta_{0}$,

$$
\begin{aligned}
\int_{0}^{1} \cos \left(\theta_{p}(t)-\theta_{0}\right)|\dot{p}(t)| d t & =\int_{0}^{1} \dot{p}_{1}(t) \cos \theta_{0}+\dot{p}_{2}(t) \sin \theta_{0} d t \\
& =\cos \theta_{0}+\sin \theta_{0}=\sqrt{2} \cos \left(\theta_{0}-\pi / 4\right) .
\end{aligned}
$$

Thus (5.4) collapses to $\tilde{Q}(p)-\sqrt{2}$, where

$$
\tilde{Q}(p):=\ell\left(\Sigma^{\operatorname{int}}(p)\right):=\ell_{p},
$$

the length of the internal h-bimorphism $\Sigma^{\mathrm{int}}(p)$ parametrized by $p$. Therefore for any $\varepsilon$ the functional $\tilde{J}+\varepsilon \tilde{Q}_{\text {temp }}$ differs from $\tilde{J}+\varepsilon \tilde{Q}$ only by an additive constant.

It is nonetheless instructive to consider the formula (5.4). First, observe that $1-\cos (\theta-\pi / 4)$ is in some sense a proxy for the small-deviation approximation $\frac{1}{2}(\theta-\pi / 4)^{2}$, but unlike the approximation it extends continuously from $\widetilde{\mathcal{B}}^{\text {int }}$ to all of $\widetilde{\mathcal{M}}^{\text {int }}$. Second, observe that for any $C^{2}$ function $g: \mathbf{R} /(2 \pi \mathbf{Z}) \rightarrow \mathbf{R}$, from (5.1) and (5.2) we have

$\delta \int_{0}^{1} g(\theta)|\dot{p}| d t=\int_{0}^{1}\left(g^{\prime}(\theta) \dot{f}-g(\theta) \dot{\theta} f+\frac{d}{d t}(\lambda g(\theta))\right) d t=-\int_{0}^{1}\left(g^{\prime \prime}(\theta)+g(\theta)\right) \dot{\theta} f d t$.

The case $g \equiv 1$ yields the simple formula

$$
\left.D \tilde{Q}\right|_{p}\left(f \mathbf{N}_{p}+\lambda \mathbf{T}_{p}\right)=\delta \tilde{Q}=-\int_{0}^{1} \dot{\theta}_{p} f d t,
$$

and only functions $g$ for which $g^{\prime \prime}+g \equiv 1$ have such a simple variation. Thus the only functionals of the form $p \mapsto \int_{0}^{1} g\left(\theta_{p}\right)|\dot{p}| d t$ having the simple variational formula (5.7) are those of the form (5.4) with " $\cos \left(\theta_{p}(t)-\pi / 4\right)$ " replaced with 
" $A \cos \left(\theta_{p}(t)-\theta_{0}\right)$ " for arbitrary constants $A, \theta_{0}$. But from the above, such a functional differs from $\tilde{Q}$ only by the constant $A \sqrt{2} \cos \left(\theta_{0}-\pi / 4\right)$, and hence are all equivalent to $\tilde{Q}$ for variational purposes.

These considerations motivate the choice of the simple functional $\varepsilon \tilde{Q}$ as our perturbation (which, as we shall see, acts as a regularization term):

Definition 5.1. Given $\varepsilon>0$, the ( $\left.\varepsilon_{-}\right)$regularized grand objective functional $\tilde{J}^{\varepsilon}$ : $\widetilde{\mathcal{M}}=\widetilde{\mathcal{M}}^{\text {int }} \times \widetilde{\mathcal{K}} \times \widetilde{\mathcal{K}} \rightarrow \mathbf{R}$ is defined by

$$
\tilde{J}^{\varepsilon}\left(p ; \kappa_{1}, \kappa_{2}\right)=\tilde{J}\left(p ; \kappa_{1}, \kappa_{2}\right)+\varepsilon \tilde{Q}(p) .
$$

Similarly we define regularized objective functionals $\widetilde{J}^{c, \varepsilon}: \widetilde{\mathcal{M}}^{\text {int }, c} \rightarrow \mathbf{R}, J^{\varepsilon}: \mathcal{M}^{\text {int }} \rightarrow$ $\mathbf{R}$ by adding $\varepsilon \tilde{Q}$ (respectively $\varepsilon Q$ ) to $\tilde{J}^{c}$ (resp. $J$ ). Holding $\kappa_{1}, \kappa_{2}$ fixed, we obtain the regularized objective functionals $\tilde{J}_{\left(\kappa_{1}, \kappa_{2}\right)}^{\varepsilon}$ on $\widetilde{\mathcal{M}}^{\text {int }}, \widetilde{J}_{\left(\kappa_{1}, \kappa_{2}\right)}^{c, \varepsilon}$ on $\widetilde{\mathcal{M}}^{\text {int,c }}$, and $J_{\left(\kappa_{1}, \kappa_{2}\right)}^{\varepsilon}$ on $\mathcal{M}^{\text {int }}$. If $C_{i, b}, i=1,2$, are based plane curves whose normalized curvature functions are $\kappa_{i}$, we also write $\tilde{J}_{\left(C_{1, b}, C_{2, b}\right)}^{\varepsilon}:=\tilde{J}_{\left(\kappa_{1}, \kappa_{2}\right)}^{\varepsilon}, J_{\left(C_{1, b}, C_{2, b}\right)}^{\varepsilon}=J_{\left(\kappa_{1}, \kappa_{2}\right)}^{\varepsilon}$.

Note that $\tilde{J}^{\varepsilon}$ is minimized at parametrized identity bimorphisms: $\tilde{J}^{\varepsilon}\left(\tau_{a}\left(p^{\Delta}\right) ; \kappa, \kappa\right)$ $=0$. In particular if $C_{1, b}=C_{2, b}$ then the absolute minimum of $\tilde{J}_{\left(C_{1, b}, C_{2, b}\right)}^{\varepsilon}$ is achieved at $p^{\Delta}$ and its $S^{1}$-translates.

Remark 5.2. In our notation, the regularization term used in [T] can be shown to be the parametrization-independent functional

$$
\tilde{Q}_{\mathrm{T}}(p)=2 \int_{0}^{1} \frac{\left(\dot{\theta}_{p}\right)^{2}}{|\dot{p}|} d t
$$

Like $\tilde{Q}$, this functional is minimized on $\widetilde{\mathcal{M}}^{\text {int,c }}$ if and only if $\theta_{p} \equiv \pi / 4$. However, the first variation of $\tilde{Q}_{\mathrm{T}}$ in the direction $f \mathbf{N}_{p}+\lambda \mathbf{T}_{p}$ is already second-order in $f$, one order higher than the first variations of $\tilde{J}_{\left(\kappa_{1}, \kappa_{2}\right)}^{c}$ and $\tilde{Q}$, and the second variation of $\tilde{Q}_{\mathrm{T}}$ is third-order in $f$, while the second variations of $\tilde{J}_{\left(\kappa_{1}, \kappa_{2}\right)}^{c}$ and $\tilde{Q}$ are only second-order. Thus $\tilde{Q}$ seems the more suitable regularization term in that it does not change the order of the Euler-Lagrange equation or the second-variation formula.

From (4.6) and (5.7), a point $p$ in the interior of $\widetilde{\mathcal{B}}^{\text {int,c }}$ is a critical point of $\tilde{J}_{\left(\kappa_{1}, \kappa_{2}\right)}^{\varepsilon}$ if and only if

$$
\tilde{Z}_{\kappa_{1}, \kappa_{2}}^{\varepsilon}:=\tilde{Z}_{\kappa_{1}, \kappa_{2}}-\varepsilon \dot{\theta} \equiv 0 .
$$

The vector field $\tilde{Z}_{\kappa_{1}, \kappa_{2}}^{\varepsilon}$ on $\widetilde{\mathcal{M}}^{\text {int }, c}$ is $S^{1}$-equivariant- $\tilde{Z}_{\kappa_{1}, \kappa_{2}}^{\varepsilon}\left(\tau_{a}(p)\right)=\tau_{a}\left(\tilde{Z}_{\kappa_{1}, \kappa_{2}}^{\varepsilon}(p)\right)$ and therefore descends to a vector field on $Z_{\kappa_{1}, \kappa_{2}}^{\varepsilon}$ on $\mathcal{M}^{\text {int }}$.

Note that a non-identity GEM is never a critical point of $\tilde{Z}_{\kappa_{1}, \kappa_{2}}^{\varepsilon}$, so we will restrict attention to the behavior of $Z_{\kappa_{1}, \kappa_{2}}^{\varepsilon}$ in a neighborhood of an identity GEM. This allows us to prove Theorem 5.4 below.

Notation 5.3. For any $W \subset \mathcal{B}_{\infty}^{\text {int }}$ and any $C^{\infty}$ based plane curves $C_{1, b}, C_{2, b}$, let $C_{1}, C_{2}$ denote the underlying curves and let $W\left(C_{1, b}, C_{2, b}\right)$ denote the subset of $\mathcal{B}_{\infty,+}\left(C_{1}, C_{2}\right)$ corresponding to $W$ under the homeomorphism $\mathcal{B}_{\infty,+}\left(C_{1}, C_{2}\right) \cong \mathcal{B}_{\infty}^{\text {int }}$ determined by the (basepoint-dependent) factorization (5.3) (see [G, (2.12)]).

Note that if $W=\{\Delta\}$, then $W\left(C_{1, b}, C_{2, b}\right)=\left\{\Sigma_{\mathrm{id}}\left(C_{1, b}, C_{2, b}\right)\right\}$, the pseudoidentity bimorphism defined in $\$ 1$. 
Theorem 5.4. Fix $\varepsilon>0$ and let $\kappa_{0} \in \widetilde{\mathcal{K}}_{\infty}$ be nonconstant. Then there exist $j_{1}, j_{2}<\infty$, an open $C^{j_{1}}$-neighborhood $U$ of $\kappa_{0}$ in $\widetilde{\mathcal{K}}_{\infty}$, and an open $C^{j_{2}}$ neighborhood $W$ of the identity internal bimorphism $\Delta$, such that for every pair of based curves $\left(C_{1, b}, C_{2, b}\right)$ whose normalized curvature functions $\kappa_{i}$ lie in $U$ there exists a unique internal bimorphism bimorphism $\Sigma^{\mathrm{int}}=\Sigma^{\mathrm{int}}\left(\kappa_{1}, \kappa_{2}\right)$ in $W$ that minimizes $\left.J_{\left(\kappa_{1}, \kappa_{2}\right)}^{\varepsilon}\right|_{W}$, and hence a unique $\left(C_{1}, C_{2}\right)$-bimorphism $\Sigma=\Sigma\left(C_{1, b}, C_{2, b}\right) \in$ $W\left(C_{1, b}, C_{2, b}\right)$ that minimizes $\left.J_{\left(C_{1, b}, C_{2, b}\right.}^{\varepsilon}\right|_{W\left(C_{1, b}, C_{2, b}\right)}$. The map $U \times U \rightarrow W$ defined by $\left(\kappa_{1}, \kappa_{2}\right) \mapsto \Sigma^{\text {int }}\left(\kappa_{1}, \kappa_{2}\right)$ is smooth (in fact tamely smooth).

Theorem 1.1 follows immediately from Theorem 5.4. For the meaning of "tamely smooth", see [G, §5].

The proof of Theorem 5.4 is postponed to 6 . However, we can already see why $Z_{\kappa_{1}, \kappa_{2}}^{\varepsilon}$, the $L^{2}$-gradient of the regularized objective functional, is better behaved than the unregularized functional. At the identity bimorphism $\Delta$, the tangent space $T_{\Delta} \mathcal{M}^{\text {int }}$ is naturally isomorphic to $C^{\infty}\left(S^{1}\right)$ via $\Psi_{*}: f_{1} \mapsto f_{1} \mathbf{N}_{p^{\Delta}}$ (see [G], equations (2.18) and (2.22)-(2.23)); at the point $p^{\Delta} \in \widetilde{\mathcal{M}}^{\text {int }}$ we have $\dot{\theta} \equiv 0$, so the functions $\lambda_{f}$ and $\hat{\lambda}_{f}$ defined in [G, §2] all vanish. Let $\kappa_{0} \in \widetilde{\mathcal{K}}$ and let $G_{\kappa_{0}}=Z_{\kappa_{0}, \kappa_{0}}^{\varepsilon} \circ \Psi_{*}$. An easy computation shows that

$$
A_{0}\left(f_{1}\right):=\left.D G_{\kappa_{0}}\right|_{0}\left(f_{1}\right)=-\frac{1}{\sqrt{2}}\left(\Gamma^{\prime \prime}(0) k\left(k f_{1}\right)^{*}+\varepsilon \ddot{f}_{1}\right)
$$

where $k=\sqrt{2} \kappa_{0}$. Taking the $L^{2}$ inner-product of $A_{0}\left(f_{1}\right)$ with $f_{1}$, we have

$$
\sqrt{2}\left\langle A_{0}\left(f_{1}\right), f_{1}\right\rangle_{L^{2}}=2 \Gamma^{\prime \prime}(0)\left\|\left(\kappa_{0} f_{1}\right)^{\cdot}\right\|_{L^{2}}^{2}+\varepsilon\left\|\dot{f}_{1}\right\|_{L^{2}}^{2} .
$$

Hence if $\kappa_{0}$ is nonconstant then the elliptic self-adjoint operator $A_{0}$ has only trivial kernel. (If $\kappa_{0}$ is constant - i.e. if the corresponding plane curve is a perfect circle - then $A_{0}$ has a 1-dimensional kernel.) Since elliptic operators on compact manifolds have discrete spectra, it follows that in the non-circular case there exists $c=c\left(\kappa_{0}, \varepsilon\right)>0$ such that

$$
\left\langle A_{0}(f), f\right\rangle_{L^{2}} \geq c\|f\|_{L^{2}}^{2} .
$$

This (together with elliptic regularity; cf. [GT]) implies that $\left.D G_{\kappa_{0}}\right|_{0}$ is a topological isomorphism $C^{\infty}\left(S^{1}\right) \rightarrow C^{\infty}\left(S^{1}\right)$. Both on Banach spaces and Fréchet spaces, a necessary starting point for any inverse-function theorem on a neighborhood of a point $x_{0}$ is that the derivative at $x_{0}$ be a topological isomorphism. Without the regularization term, if $\kappa_{0}$ vanished anywhere then $A_{0}$ would not be elliptic or surjective, its image missing any function that fails to vanish at the zeroes of $\kappa_{0}$. Thus $D G_{\kappa_{0}}$ would not be an isomorphism at 0 , the point corresponding to the identity internal bimorphism.

To apply the Tame Implicit Function Theorem [G, Theorem 5.3], we must show that, extending $G_{\kappa_{0}}$ to the function $G: \mathcal{M}^{\text {int }} \times \widetilde{\mathcal{K}} \times \widetilde{\mathcal{K}} \rightarrow C^{\infty}\left(S^{1}\right)$ defined by $G\left(f, \kappa_{1}, \kappa_{2}\right)=Z_{\kappa_{1}, \kappa_{2}}^{\varepsilon} \circ \Psi_{*}$, the derivative of $G$ with respect to the first variable remains an isomorphism for all points $\left(p, \kappa_{1}, \kappa_{2}\right)$ in a sufficiently small neighborhood of $\left(p^{\Delta}, \kappa_{0}, \kappa_{0}\right)$, and that the family of inverses of the derivative is tame. This is much of what is proved in $\sqrt{6}$, the remainder of the work in that section is devoted to proving that the critical points produced this way - the zeroes of $G_{\kappa_{0}}$-are in fact minima of the corresponding objective functionals on suitable neighborhoods.

Note that in contrast to the unregularized case, in Theorem 5.4 we obtain local uniqueness for non-circular convex curves as well as for nonconvex curves. Of course 
if $C_{1}$ or $C_{2}$ is a circle there can never be a unique optimal $\left(C_{1}, C_{2}\right)$-bimorphism, so there is no hope of removing the " $\kappa_{0}$ nonconstant" hypothesis from Theorem 5.4.

\section{The Proof of Theorem 5.4}

In this section we abbreviate the $C^{n}$ norms on $C^{\infty}\left(S^{1}\right)$ and $C^{\infty}\left(S^{1}, \mathbf{R}^{2}\right)$ as \|\|$_{n}$.

Before proceeding to the proof of Theorem 5.4 we lay some groundwork. For $f \in C^{\infty}\left(S^{1}\right)$ let $\|f\|_{L_{n}^{2}}=\left(\sum_{i=0}^{n}\left\|f^{(i)}\right\|_{L^{2}}^{2}\right)^{1 / 2}$, the standard $n^{\text {th }}$-order Sobolev norm. For use in the lemma below, we recall the dimension-1 Sobolev inequality (see $[\mathrm{A}$ ): for all $n \geq 0$ and all $f \in C^{\infty}\left(S^{1}\right)$ (more generally all $f$ in the Sobolev space $\left.L_{n+1}^{2}\left(S^{1}\right)\right)$, there exists a constant $C$ such that

$$
\|f\|_{n} \leq C\|f\|_{L_{n+1}^{2}} .
$$

We will also use the following interpolation inequalities for functions $f, g \in C^{\infty}\left(S^{1}\right)$ (see [H] Corollaries II.2.2.2 and II.2.2.3, p. 144]):

$$
\begin{aligned}
\|f\|_{m}\|g\|_{n} & \leq C(m, n)\left(\|f\|_{m+n-1}\|g\|_{1}+\|f\|_{1}\|g\|_{m+n-1}\right) \\
\|f g\|_{n} & \leq C(n)\left(\|f\|_{n}\|g\|_{0}+\|f\|_{0}\|g\|_{n}\right) .
\end{aligned}
$$

We will need several technical lemmas.

Lemma 6.1. For all $n \geq 1$, there exists a constant $C$ such that for all $h, f \in$ $C^{\infty}\left(S^{1}\right)$ (in fact for all $h, f \in L_{\max \{n, 2\}}^{2}\left(S^{1}\right)$ ),

$$
\|h f\|_{L_{n}^{2}} \leq C\left(\|h\|_{n}\|f\|_{L_{2}^{2}}+\|h\|_{1}\|f\|_{L_{n}^{2}}\right) .
$$

For $n=1$ the first term on the right-hand side can be omitted, while for $n=2$ the second term is redundant.

Proof. For $n=1$ the bound $C\|h\|_{1}\|f\|_{L_{1}^{2}}$ is obvious. For $n \geq 1$ we have

$$
\begin{aligned}
\left\|(h f)^{(n+1)}\right\|_{L^{2}} & \leq C\left(\left\|h^{(n+1)} f\right\|_{L^{2}}+\sum_{j=1}^{n+1}\left\|h^{(n+1-j)} f^{(j)}\right\|_{L^{2}}\right) \\
& \leq C\left(\left\|h^{(n+1)} f\right\|_{L^{2}}+\sum_{j=1}^{n+1}\left\|h^{(n+1-j)} f^{(j)}\right\|_{0}\right) .
\end{aligned}
$$

The result now follows by induction, using (6.2) and (6.1).

Lemma $6.2([\mathrm{G}]$, Lemma 4.4). Let $\alpha<1$. For all $n \geq 1$ there exists a constant $C$ such that for all $c_{1}>0$ and all $h \in C^{\infty}\left(S^{1}\right)$ satisfying $h(t) \geq c_{1}$ for all $t \in S^{1}$, we have

$$
\left\|h^{\alpha}\right\|_{n} \leq\left\|h^{\alpha}\right\|_{0}+C c_{1}^{\alpha-n}\|h\|_{0}^{n-1}\left\|h-c_{1}\right\|_{n} .
$$

Corollary 6.3. Let $\alpha<1$. For all $n \geq 1$ there exists a constant $C$ such that for all $c_{1}>0$ and all $g \in C^{\infty}\left(S^{1}\right)$,

$$
\left\|\left(g^{2}+c_{1}^{2}\right)^{\alpha}\right\|_{n} \leq c_{1}^{2 \alpha}+C\left(c_{1}^{2 \alpha-2}\|g\|_{0}+c_{1}^{2 \alpha-2 n}\|g\|_{0}^{n-1}\right)\|g\|_{n}+ \begin{cases}\|g\|_{0}^{2 \alpha} & \text { if } \alpha>0 \\ 0 & \text { if } \alpha<0\end{cases}
$$


Proof. Let $h=g^{2}+c_{1}^{2}, h_{1}=g^{2}$. For all $n \geq 1, h_{1}^{(n)}=\sum_{i=0}^{n}\left(\begin{array}{c}n \\ i\end{array}\right) g^{(i)} g^{(n-i)}$. Hence from [G, inequality (4.76)] we have

$$
\left\|h^{(n)}\right\|_{0} \leq C \sum_{i=0}^{r}\|g\|_{i}\|g\|_{n-i} \leq C\|g\|_{0}\|g\|_{n}
$$

The inequality (6.4) now follows from Lemma 6.2

We will also need the following lemma to ensure that a critical point $p$ of $J_{\left(\kappa_{1}, \kappa_{2}\right)}^{\varepsilon}$ with strongly positive-definite Hessian, with respect to the $L_{1}^{2}$-norm, is a local minimizer of $J_{\left(\kappa_{1}, \kappa_{2}\right)}^{\varepsilon}$. This is not implied by Lemma 3.1 because at the putative minima $p$ we will produce, we do not have a lower bound on $\left.H J_{\left(\kappa_{1}, \kappa_{2}\right)}^{\varepsilon}\right|_{p}(X, X)$ of the form $c\|X\|_{C^{1}}^{2}$, but only one of the form $c\|X\|_{L_{1}^{2}}^{2}$; cf. (3.11). After the lemma below we will resume assuming that $\Gamma$ is $C^{\infty}$, but we state the lemma in greater generality.

Lemma 6.4. Assume the function $\Gamma$ defining the objective functionals is $C^{2}$. Let $\left(p_{0}, \kappa_{1,0}, \kappa_{2,0}\right) \in B_{0} \times B_{1} \times B_{2} \subset \widetilde{\mathcal{M}}^{\text {int }, c} \times \widetilde{\mathcal{K}} \times \widetilde{\mathcal{K}}$, where $B_{0}$ is contained in a $C^{2}$-ball of radius $<\ell_{p_{0}}$ centered at $p_{0}$ and where $B_{1}, B_{2}$ are bounded sets in the $C^{2}$-norm. Then for all $\epsilon>0$ there exists $\epsilon^{\prime}>0$ such that if $\left(p, \kappa_{1}, \kappa_{2}\right) \in B_{0} \times B_{1} \times B_{2}$ and $X \in C^{\infty}\left(S^{1}, \mathbf{R}^{2}\right)$ with $\|X\|_{1}<\epsilon^{\prime}$, then

$$
\left|\tilde{J}_{\left(\kappa_{1}, \kappa_{2}\right)}^{\varepsilon}(p+X)-\tilde{J}_{\left(\kappa_{1}, \kappa_{2}\right)}^{\varepsilon}(p)-D \tilde{J}_{\left(\kappa_{1}, \kappa_{2}\right)}^{\varepsilon}\right|_{p}(X)-\left.\frac{1}{2} H \tilde{J}_{\left(\kappa_{1}, \kappa_{2}\right)}^{\varepsilon}\right|_{p}(X, X) \mid \leq \epsilon\|X\|_{L_{1}^{2}}^{2} .
$$

Proof. From Lemma 3.1, $\tilde{J}_{\left(\kappa_{1}, \kappa_{2}\right)}$ is $C^{2}$, so the derivative and Hessian written in (6.5) make sense. Let $B_{r}^{0}$ be the ball of radius $r$ in $C^{0}\left(S^{1}\right)$ centered at 0 , and in the spirit of the proof of Lemma 3.1 define $F: C^{0}\left(S^{1}\right) \oplus C^{0}\left(S^{1}\right) \oplus C^{0}\left(S^{1}\right) \rightarrow C^{0}\left(S^{1}\right)$ by $F\left(h_{1}, h_{2}, h_{3}\right)=\left(\Gamma \circ h_{1}\right) h_{2}+h_{3}$.

Let $\epsilon>0$. Since $\Gamma$ is $C^{2}$, so is $F$; thus, given $\epsilon_{1}>0$ there exists $\epsilon_{2}=\epsilon_{2}(r)>0$ such that for every triple of functions $h=\left(h_{1}, h_{2}, h_{3}\right) \in B_{r}^{0} \times B_{r}^{0} \times B_{r}^{0}$ and all $\zeta=\left(\zeta_{1}, \zeta_{2}, \zeta_{3}\right) \in C^{0}\left(S^{1}\right) \oplus C^{0}\left(S^{1}\right) \oplus C^{0}\left(S^{1}\right)$ with $\|\zeta\|_{0}<\epsilon_{2}$,

$$
\left\|F(h+\zeta, h+\zeta)-\left.D F\right|_{h}(\zeta)-\left.\frac{1}{2} H F\right|_{h}(\zeta, \zeta)\right\|_{0} \leq \epsilon_{1}\|\zeta\|_{0}^{2}
$$

Now let $\left(p, \kappa_{1}, \kappa_{2}\right) \in B_{0} \times B_{1} \times B_{2}$ and let $h_{1}=\hat{\phi}_{\left(p, \kappa_{1}, \kappa_{2}\right)}, h_{2}=|\dot{p}|$, and $h_{3}=$ $\varepsilon\left(1-\cos \circ\left(\theta_{p}-\pi / 4\right)\right)|\dot{p}|$; our hypotheses ensure that $h_{1}, h_{2}, h_{3} \in B_{r}^{0}$ for some finite $r$. For $X \in C^{\infty}\left(S^{1}\right)$ consider $\left.\zeta_{1}=\hat{\phi}_{\left(p+X, \kappa_{1}, \kappa_{2}\right)}-\hat{\phi}_{\left(p, \kappa_{1}, \kappa_{2}\right)}, \zeta_{2}=\mid(p+X)\right)^{\dot{*}} \mid-$ $|\dot{p}|$, and $\zeta_{3}=\left(1-\cos \circ\left(\theta_{(p+X)}-\pi / 4\right)\right)\left|(p+X)^{\bullet}\right|-\left(1-\cos \circ\left(\theta_{p}-\pi / 4\right)\right)|\dot{p}|$. The restriction on the radius of $C^{2}$-radius (hence $C^{1}$-radius) of $B_{0}$ ensures a uniform lower bound on $|\dot{p}(t)|$ for all $p \in B_{0}, t \in S^{1}$. Together with the $C^{2}$-boundedness of $B_{1}, B_{2}$, this implies the uniform pointwise estimates $\left|\zeta_{2}(t)\right| \leq C\left(B_{0}\right)|\dot{X}(t)|^{2}$, $\left|\zeta_{3}(t)\right| \leq \varepsilon C\left(B_{0}\right)|\dot{X}(t)|^{2}$, and $\left|\zeta_{1}(t)\right| \leq C\left(B_{0}, B_{1}, B_{2}\right)\left(|X(t)|^{2}+|\dot{X}(t)|^{2}\right)$. Hence there exists $\epsilon_{3}=\epsilon_{3}\left(B_{1}, B_{2}, B_{3}\right)>0$ such that if $\|X\|_{1}<\epsilon_{3}$, then $\|\zeta\|_{0} \leq \epsilon_{2}$, and 
hence the left-hand side of (6.6) is $\leq \epsilon_{1}\|X\|_{1}^{2}$. Since

$$
\begin{aligned}
\tilde{J}_{\left(\kappa_{1}, \kappa_{2}\right)}^{\varepsilon}(p+X)-\tilde{J}_{\left(\kappa_{1}, \kappa_{2}\right)}^{\varepsilon}(p)-\left.D \tilde{J}_{\left(\kappa_{1}, \kappa_{2}\right)}^{\varepsilon}\right|_{p}(X)-\left.\frac{1}{2} H \tilde{J}_{\left(\kappa_{1}, \kappa_{2}\right)}^{\varepsilon}\right|_{p}(X, X) \\
=\int_{0}^{1}\left(F(h+\zeta, h+\zeta)-\left.D F\right|_{h}(\zeta)-\left.\frac{1}{2} H F\right|_{h}(\zeta, \zeta)\right) d t,
\end{aligned}
$$

(6.5) follows.

Note that the lemma above does not imply the (false) statement that $\tilde{J}_{\left(\kappa_{1}, \kappa_{2}\right)}$ or $\tilde{J}_{\left(\kappa_{1}, \kappa_{2}\right)}^{\varepsilon}$ extends to a $C^{2}$ (or even $C^{1}$ ) functional on the space of $L_{1}^{2}$ internal h-bimorphisms.

In order to prove Theorem 5.4 we will need a formula for the derivative of $\left.\tilde{Z}_{\kappa_{1}, \kappa_{2}}^{\varepsilon}\right|_{\widetilde{\mathcal{M}}^{\text {int }, c}}$; we obtain this by varying $\tilde{Z}_{\kappa_{1}, \kappa_{2}}^{\varepsilon}$ at $p \in \widetilde{\mathcal{M}}^{\text {int }, c}$ in a direction $f \mathbf{N}_{p}+$ $\lambda \mathbf{T}_{p} \in T_{p} \widetilde{\mathcal{M}}^{\mathrm{int}, c}$, where $\lambda=\hat{\lambda}_{p}(f)+a$ for some $a \in \mathbf{R}$. Using (5.1)-(5.2) and (4.2), we find

$$
\begin{aligned}
\left.D \tilde{Z}_{\kappa_{1}, \kappa_{2}}^{\varepsilon}\right|_{p}\left(f \mathbf{N}_{p}+\lambda \mathbf{T}_{p}\right)=\ell_{p}^{-1}\{- & k\left[\left(\Gamma^{\prime \prime} \circ \hat{\phi}\right)(k f)^{\circ}\right]^{\cdot}+((A \circ \hat{\phi}) \dot{f})^{\cdot} \\
+ & f\left[\left(\Gamma^{\prime \prime} \circ \hat{\phi}\right)(\hat{\phi} \dot{\theta})^{2}\right. \\
& +\left(\Gamma^{\prime} \circ \hat{\phi}\right)^{\cdot}\left((\hat{\phi})^{\cdot}+k \dot{\theta}+\ell_{p}\left(k_{2}^{\prime}-k_{1}^{\prime}\right)\right) \\
& \left.+k\left(\left(A^{\prime} \circ \hat{\phi}\right) \dot{\theta}\right)^{\cdot}-\dot{k}\left(A^{\prime} \circ \hat{\phi}\right) \dot{\theta}\right] \\
+ & \left.\left(\lambda \tilde{Z}_{\kappa_{1}, \kappa_{2}}^{\varepsilon}\right)^{\cdot}-\varepsilon \ddot{f}\right\},
\end{aligned}
$$

where $\hat{\phi}=\hat{\phi}_{\left(p, \kappa_{1}, \kappa_{2}\right)}$ etc. Using this we prove one last lemma before proceeding to the main proof.

Lemma 6.5. Let $\kappa_{0} \in \widetilde{\mathcal{K}}$ be nonconstant. Then there exists $\epsilon_{2}=\epsilon_{2}\left(\kappa_{0}, \varepsilon\right)>0$ and $c_{1}\left(\kappa_{0}, \varepsilon\right)>0$ such that if $\left(p, \kappa_{1}, \kappa_{2}\right) \in \widetilde{\mathcal{M}}^{\text {int }, c} \times \widetilde{\mathcal{K}} \times \widetilde{\mathcal{K}}$ with $\left\|\kappa_{i}-\kappa_{0}\right\|_{1}<\epsilon$ and $\left\|p-p^{\Delta}\right\|_{3}<\epsilon$, and if $p$ is a critical point of $\tilde{J}_{\left(\kappa_{1}, \kappa_{2}\right)}^{c, \varepsilon}$, then for all $f, \lambda \in C^{\infty}\left(S^{1}\right)$ we have

$$
\left.H \tilde{J}_{\left(\kappa_{1}, \kappa_{2}\right)}^{\varepsilon}\right|_{p}\left(f \mathbf{N}_{p}+\lambda \mathbf{T}_{p}, f \mathbf{N}_{p}+\lambda \mathbf{T}_{p}\right)=\left.H \tilde{J}_{\left(\kappa_{1}, \kappa_{2}\right)}^{c, \varepsilon}\right|_{p}\left(X_{p, f}, X_{p, f}\right) \geq c_{1}\left(\kappa_{0}\right)\|f\|_{L_{1}^{2}}^{2},
$$

where $X_{p, f}=f \mathbf{N}_{p}+\hat{\lambda}_{p}(f) \mathbf{T}_{p}$.

Proof. Let $\langle\cdot, \cdot\rangle$ denote the $L^{2}$ inner product on $C^{\infty}\left(S^{1}\right)$. From (4.1) and (5.7) at a general point $q \in \widetilde{\mathcal{M}}^{\text {int,c}}$, for any $\lambda_{1} \in C^{\infty}\left(S^{1}\right)$ we have $D \tilde{J}_{\left(\kappa_{1}, \kappa_{2}\right)}^{\varepsilon}\left(f_{1} \mathbf{N}_{q}+\lambda_{1} \mathbf{T}_{q}\right)=$ $\left.D \tilde{J}_{\left(\kappa_{1}, \kappa_{2}\right)}^{c, \varepsilon}\right|_{q}\left(X_{f, q}\right)=\left\langle\tilde{Z}_{\kappa_{1}, \kappa_{2}}^{\varepsilon}(q), f_{1}\right\rangle$. The insensitivity to $\lambda_{1}$ reflects the invariance of $\tilde{J}_{\left(\kappa_{1}, \kappa_{2}\right)}^{\varepsilon}$ under the reparametrization group $\operatorname{Diff}^{+}\left(S^{1}\right)$ and implies that a point in $\widetilde{\mathcal{M}}^{\text {int,c }}$ is critical for $\tilde{J}_{\left(\kappa_{1}, \kappa_{2}\right)}^{c, \varepsilon}$ if and only if it is critical for $\tilde{J}_{\left(\kappa_{1}, \kappa_{2}\right)}^{\varepsilon}$.

At a critical point $p$ (but not elsewhere) we may compute the Hessian of both the restricted and the non-restricted functional by differentiating the preceding equation holding $f_{1}, \lambda_{1}$ fixed, yielding

$$
\begin{aligned}
H \tilde{J}_{\left(\kappa_{1}, \kappa_{2}\right)}^{\varepsilon}\left(f_{1} \mathbf{N}_{p}+\lambda_{1} \mathbf{T}_{p}, f_{2} \mathbf{N}_{p}+\lambda_{2} \mathbf{T}_{p}\right) & =\left.H \tilde{J}_{\left(\kappa_{1}, \kappa_{2}\right)}^{c, \varepsilon}\right|_{p}\left(X_{p, f_{1}}, X_{p, f_{2}}\right) \\
& =\left\langle\left. D \tilde{Z}_{\kappa_{1}, \kappa_{2}}^{\varepsilon}\right|_{p}\left(X_{f_{2}, p}\right), f_{1}\right\rangle_{L^{2}},
\end{aligned}
$$


so using (6.7) and integrating by parts we have

(6.9)

$\left.H \tilde{J}_{\left(\kappa_{1}, \kappa_{2}\right)}^{c, \varepsilon}\right|_{p}\left(X_{p, f}, X_{p, f}\right)=\ell_{p}^{-1}\left\{\left\langle(k f)^{\cdot},\left(\Gamma^{\prime \prime} \circ \hat{\phi}\right)(k f) \dot{ }\right\rangle+\varepsilon\langle\dot{f}, \dot{f}\rangle+\langle\dot{f},(A \circ \hat{\phi}) \dot{f}\rangle+\langle f, b f\rangle\right\}$

where $b$ is the expression in square brackets multiplying $f$ in (6.7). For $\left(p, \kappa_{1}, \kappa_{2}\right)$ $C^{1}$-close to $\left(p^{\Delta}, \kappa_{0}, \kappa_{0}\right), \hat{\phi}$ is $C^{0}$-close to 0 and $\ell_{p}$ is close to $\sqrt{2}$. Hence on a small enough $C^{0}$ neighborhood of $\left(p^{\Delta}, \kappa_{0}, \kappa_{0}\right)$ we have

$$
\ell_{p}^{-1}\left(\left\langle(k f)^{\cdot},\left(\Gamma^{\prime \prime} \circ \hat{\phi}\right)(k f) \dot{\rangle}\right\rangle+\varepsilon\langle\dot{f}, \dot{f}\rangle\right) \geq \frac{1}{2}\left(\Gamma^{\prime \prime}(0)\left\langle(k f)^{\cdot},(k f)\right\rangle+\varepsilon\langle\dot{f}, \dot{f}\rangle\right) .
$$

On this neighborhood assume that $\left\|\kappa_{i}-\kappa_{0}\right\|_{1}<\epsilon \leq 1$, implying $\left\|k-\sqrt{2} \kappa_{0}\right\|_{1}<C \epsilon$. An easy computation then shows that

$$
\left\langle(k f)^{\cdot},(k f)^{\cdot}\right\rangle \geq\left\langle\left(\sqrt{2} \kappa_{0} f\right)^{\cdot},\left(\sqrt{2} \kappa_{0} f\right)^{\cdot}\right\rangle-C\left(\kappa_{0}\right) \epsilon\|f\|_{L_{1}^{2}}^{2} .
$$

Let $A_{0}^{\prime}$ be the operator $f \mapsto-\frac{1}{2}\left(\Gamma^{\prime \prime}(0) \sqrt{2} \kappa_{0}\left(\sqrt{2} \kappa_{0} f_{1}\right)^{\prime \prime}+\varepsilon \ddot{f}_{1}\right)$. Just as for the operator $A_{0}$ in (5.9), $A_{0}^{\prime}$ is self-adjoint and elliptic, and has only trivial kernel. Hence there is a constant $c_{2}\left(\kappa_{0}, \varepsilon\right)>0$ such that

$$
\frac{1}{2}\left(\left\langle\left(\sqrt{2} \kappa_{0} f\right)^{\cdot},\left(\sqrt{2} \kappa_{0} f\right)^{\dot{ }}\right\rangle+\varepsilon\langle\dot{f}, \dot{f}\rangle\right)=\left\langle f, A_{0}^{\prime} f\right\rangle \geq c_{2}\left(k_{0}, \varepsilon\right)\|f\|_{L_{1}^{2}}^{2}
$$

(see GT]). If we further assume $\left\|p-p^{\Delta}\right\|_{3}<\epsilon$ then $\|A \circ \hat{\phi}\|_{0}<C\left(\kappa_{0}\right) \epsilon$ and $\|b\|_{0}<C\left(\kappa_{0}\right) \epsilon$. Incorporating this with (6.8)-(6.12), we have

$$
\begin{aligned}
\left.H \tilde{J}_{\left(\kappa_{1}, \kappa_{2}\right)}^{c, \varepsilon}\right|_{p}\left(X_{p, f}, X_{p, f}\right) & \geq\left(c_{2}\left(\kappa_{0}, \varepsilon\right)-C\left(\kappa_{0}\right) \epsilon\right)\|f\|_{L_{1}^{2}}^{2} \\
& \geq \frac{1}{2} c_{2}\left(\kappa_{0}, \varepsilon\right)\|f\|_{L_{1}^{2}}^{2}
\end{aligned}
$$

if we take $\epsilon$ small enough.

Proof of Theorem [5.4. We will apply the Tame Implicit Function Theorem [G, Theorem 5.3]. For this, we view the curvature functions $\kappa_{1}, \kappa_{2}$ in (5.8) as variables, and $Z^{\varepsilon}:=Z-\varepsilon \dot{\theta}$ as a map $\widetilde{\mathcal{M}}=\mathcal{M}^{\text {int }} \times \widetilde{\mathcal{K}} \times \widetilde{\mathcal{K}} \rightarrow C^{\infty}\left(S^{1}\right)$ induced by the map $\tilde{Z}^{\varepsilon}: \widetilde{\mathcal{M}}^{\text {int }} \times \widetilde{\mathcal{K}} \times \widetilde{\mathcal{K}} \rightarrow C^{\infty}\left(S^{1}\right)$. Since the formula for $\tilde{J}$ makes perfectly good sense for $\kappa_{1}, \kappa_{2} \in C^{\infty}\left(S^{1}\right)$ (not just for $\kappa_{i} \in \widetilde{\mathcal{K}}$ ), it suffices to prove Theorem 5.4 with the domain of $\tilde{J}^{\varepsilon}$ extended to $\mathcal{M}^{\text {int }} \times C^{\infty}\left(S^{1}\right) \times C^{\infty}\left(S^{1}\right)$; we do this henceforth.

We examine $Z^{\varepsilon}$ in a neighborhood of an identity bimorphism $\left(\Delta, \kappa_{0}, \kappa_{0}\right)$. Using the atlas for $\mathcal{M}^{\text {int }}$ discussed in G, Remark 4.2], one chart-representative of $Z^{\varepsilon}$ near $\left(\Delta, \kappa_{0}, \kappa_{0}\right)$ is the map $G^{\varepsilon}=\tilde{Z}^{\varepsilon} \circ \Psi_{p^{\Delta}}^{c}: V \times U \times U \rightarrow C^{\infty}\left(S^{1}\right)$, where $V$ and $U$ are small open neighborhoods of 0 and $\kappa_{0}$, respectively, in $C^{\infty}\left(S^{1}\right)$, and where, as in the proof of [G, Theorem 2.8], $\Psi_{p}^{c}(f)=\pi_{\widetilde{\mathcal{M}} \rightarrow \widetilde{\mathcal{M}}^{c}}\left(p+f \mathbf{N}_{p}+\hat{\lambda}_{p}(f)\right)$ for $p \in \widetilde{\mathcal{M}}^{\text {int }, c}$. Using (4.3), G, equations (2.16) and (2.17)], and the type of argument used in the proof of $\left[\mathrm{G}\right.$, Theorem 2.8(d)] to show that the map $G_{2}$ defined therein is tamely smooth, it is straightforward to show that $\tilde{Z}^{\varepsilon}$ is tamely smooth, and hence so is $G^{\varepsilon}$.

We will need to compute and invert $D^{(1)} G^{\varepsilon}$, the derivative of $G^{\varepsilon}$ with respect to the variable in $V$. This derivative can be computed by differentiating the restriction of $\tilde{Z}_{\kappa_{1}, \kappa_{2}}^{\varepsilon}$ to the "slice" $\mathcal{H}^{c}=\mathcal{H}_{p \Delta}^{c}$ of the bundle $\widetilde{\mathcal{M}}^{\text {int }, c} \rightarrow \mathcal{M}^{\text {int }}$. Let $\kappa_{1}, \kappa_{2}$ be the normalized curvature functions of the based curves $C_{1}, C_{2}$. For $p=\Psi_{p^{\Delta}}^{c}(f) \in \mathcal{H}^{c}$ 
and $f_{2} \mathbf{N}_{p}+\lambda \mathbf{T}_{p} \in T_{p}\left(\mathcal{H}^{c}\right)$, where $\lambda=\hat{\lambda}_{p}\left(f_{2}\right)+a_{2}$ for some $a_{2} \in \mathbf{R}$, from (6.7) we have

$$
\begin{aligned}
& L_{f, \kappa_{1}, \kappa_{2}}\left(\begin{array}{c}
f_{2} \\
\lambda
\end{array}\right):=\left.D \tilde{Z}_{\kappa_{1}, \kappa_{2}}^{\varepsilon}\right|_{p}\left(f_{2} \mathbf{N}_{p}+\lambda \mathbf{T}_{p}\right) \\
& =\ell_{p}^{-1}\left\{-\left(\Gamma^{\prime \prime} \circ \hat{\phi}\right) k\left(k f_{2}\right)^{*}-\varepsilon \ddot{f}_{2}+A(\hat{\phi}) \ddot{f}_{2}\right. \\
& +b_{1}\left(p, \kappa_{1}, \kappa_{2}\right) \dot{f}_{2}+b_{0}\left(p, \kappa_{1}, \kappa_{2}\right) f_{2}+b_{-1}\left(p, \kappa_{1}, \kappa_{2}\right) \lambda \\
& \left.+a_{-1}\left(p, \kappa_{1}, \kappa_{2}\right)\left\langle f_{2} \dot{\theta}\right\rangle\right\}
\end{aligned}
$$

where $\theta, \hat{\phi}, k$, and $k_{i}=\kappa_{i}^{\prime} \circ p_{i}$ are computed at $\left(p, \kappa_{1}, \kappa_{2}\right)$, and where the $b_{i}\left(p, \kappa_{1}, \kappa_{2}\right)$ and $a_{-1}\left(p, \kappa_{1}, \kappa_{2}\right)=-\tilde{Z}_{\kappa_{1}, \kappa_{2}}^{\varepsilon}$ are polynomials in $\Gamma^{(j)} \circ \hat{\phi}(0 \leq j \leq 3), \hat{\phi},(\hat{\phi}), \dot{\theta}, \ddot{\theta}, k^{(j)}$, $(0 \leq j \leq 2), k_{2}^{\prime}-k_{1}^{\prime}$, and $\varepsilon$. These polynomials vanish identically if $\left(p, \kappa_{1}, \kappa_{2}\right)=$ $\left(p^{\Delta}, \kappa_{0}, \kappa_{0}\right)$, in view of (3.3) and the fact that $\hat{\phi}_{\left(p^{\Delta}, \kappa_{0}, \kappa_{0}\right)} \equiv 0 \equiv \dot{\theta}_{p^{\Delta}}$. Thus, at $\left(p^{\Delta}, \kappa_{0}, \kappa_{0}\right)$, where $\ell_{p}=\sqrt{2}$ and $k=\sqrt{2} \kappa_{0}$, the right-hand side of (6.13) reduces to

$$
A_{0, \kappa_{0}}\left(f_{2}\right):=-\sqrt{2}\left(\Gamma^{\prime \prime}(0) \kappa_{0}\left(\kappa_{0} f_{2}\right)^{*}+\varepsilon \ddot{f}_{2}\right) .
$$

To differentiate $G^{\varepsilon}$ near the point $\left(0, \kappa_{0}, \kappa_{0}\right)$, we write $p=q(f) \circ \sigma_{q(f)}$, where $q(f)=p^{\Delta}+f \mathbf{N}_{p^{\Delta}}$, and take $f_{2} \mathbf{N}_{p}+\lambda \mathbf{T}_{p}=\left.D \Psi_{p^{\Delta}}^{c}\right|_{f}\left(f_{1}\right)$ (more precisely, $\left.D\left(\theta_{p}^{-1} \circ \Psi_{p^{\Delta}}^{c}\right)\right|_{f}\left(f_{1}\right)$ in the notation of the proof of [G, Theorem 2.8]). Define $M_{f}: C^{\infty}\left(S^{1}\right) \rightarrow C^{\infty}\left(S^{1}\right)$ by

$$
M_{f}\left(f_{1}\right):=\left(\begin{array}{c}
\sqrt{2}\left(2+\dot{f}^{2}\right)^{-1 / 2} f_{1} \\
\hat{\lambda}_{q(f)}\left(\sqrt{2}\left(2+\dot{f}^{2}\right)^{-1 / 2} f_{1}\right)+c\left(f ; f_{1}\right)
\end{array}\right),
$$

where

$$
\left.c\left(f ; f_{1}\right)=\int_{0}^{1} \dot{f}(t)\left\{\ell_{p}^{-1} f_{1}(t)+\left(\sigma_{q(f)}^{-1}(t)-t\right)\right) \frac{\dot{f}_{1}}{\sqrt{2+\dot{f}^{2}}}(t)\right\} d t .
$$

Let $\hat{L}_{\left(f, \kappa_{1}, \kappa_{2}\right)}(v)=L\left(v \circ \sigma_{q(f)}\right) \circ \sigma_{q(f)}^{-1}$ and define $A_{f, \kappa_{1}, \kappa_{2}}:=\hat{L}_{\left(f, \kappa_{1}, \kappa_{2}\right)} \circ M_{f}$ : $C^{\infty}\left(S^{1}\right) \rightarrow C^{\infty}\left(S^{1}\right)$. From [G, equations (4.68), (4.71), (4.75), and (4.10)], using the fact that $\lambda_{p} \Delta \equiv 0$ we then find that

$$
\left.D^{(1)} G^{\varepsilon}\right|_{\left(f, \kappa_{1}, \kappa_{2}\right)}\left(f_{1}\right)=L_{f, \kappa_{1}, \kappa_{2}}\left(M_{f}\left(f_{1}\right) \circ \sigma_{q(f)}\right)=\left(A_{f, \kappa_{1}, \kappa_{2}}\left(f_{1}\right)\right) \circ \sigma_{q(f)} .
$$

In particular, if $f=0$, then $M_{f}\left(f_{1}\right)=\left(\begin{array}{c}f_{1} \\ 0\end{array}\right)$ and $\sigma_{q(f)}=$ id. Hence $\left.D^{(1)} G^{\varepsilon}\right|_{\left(0, \kappa_{0}, \kappa_{0}\right)}$ $=A_{0, \kappa_{0}}$, which, as discussed in $\$ 5$ is an invertible elliptic operator.

Our strategy for showing invertibility of $D^{(1)} G^{\varepsilon}$ at points close to $\left(0, \kappa_{0}, \kappa_{0}\right)$, and tameness of the family of inverses, will be similar to the one used in the proof of G. Theorem 2.8(d)], but this time the nature of the operators involved forces us to use elliptic bootstrapping instead of direct $C^{j}$-bootstrapping. Let $U^{\prime}=\left\{\kappa-\kappa_{0} \mid\right.$ $\kappa \in U\}$, and define the following norms on $V \times U^{\prime} \times U^{\prime}$ :

$$
\left\|\left(f, \zeta_{1}, \zeta_{2}\right)\right\|_{n}=\|f\|_{n}+\left\|\zeta_{1}\right\|_{n-1}+\left\|\zeta_{2}\right\|_{n-1}, \quad n \geq 1 .
$$

Also, for $0<\epsilon \leq 1$ let

$$
W_{\epsilon}=\left\{\left(f, \kappa_{1}, \kappa_{2}\right) \in V \times U \times U \mid\left\|\left(f, \kappa_{1}-\kappa_{0}, \kappa_{2}-\kappa_{0}\right)\right\|_{3}<\epsilon\right\} .
$$

Since $A_{0, \kappa_{0}}$ is invertible, we can write $A_{f, \kappa_{1}, \kappa_{2}}=\left(A_{f, \kappa_{1}, \kappa_{2}} \circ A_{0, \kappa_{0}}^{-1}\right) \circ A_{0, \kappa_{0}}$ and examine the invertibility of $A_{f, \kappa_{1}, \kappa_{2}} \circ A_{0, \kappa_{0}}^{-1}$. Computing the first two derivatives of 
the first component of $M_{f}\left(f_{1}\right) \circ \sigma_{q(f)}$ and plugging into (6.13), we find, after some algebra, that $A_{f, \kappa_{1}, \kappa_{2}}\left(f_{1}\right)$ can be written in the following form:

$$
\begin{aligned}
A_{f, \kappa_{1}, \kappa_{2}}\left(f_{1}\right)= & A_{0, \kappa_{0}}\left(f_{1}\right)+P_{2} \ddot{f}_{1}+P_{1} \dot{f}_{1}+P_{0} f_{1} \\
& +P_{-1}\left\{\hat{\lambda}_{q}\left(\left(2+\dot{f}^{2}\right)^{-1 / 2} f_{1}\right)+c\left(f ; f_{1}\right)\right\} \\
& +\hat{P}_{0}\left\langle\left(2+\dot{f}^{2}\right)^{-1 / 2} f_{1} \dot{\theta}_{q}\right\rangle,
\end{aligned}
$$

where $q=q(f)$ and where the $P_{i}$ and $\hat{P}_{0}$ are universal polynomial expressions in the variables $\Gamma^{(j)} \circ \hat{\phi}(0 \leq j \leq 3), \hat{\phi},(\hat{\phi})^{\cdot}, \dot{\theta}_{q}, \ddot{\theta}_{q}, k^{(j)}, \kappa_{0}^{(j)}(0 \leq j \leq$ $2), k_{1}^{\prime}, k_{2}^{\prime}, \varepsilon, \ell_{p}, f^{(j)},(j=1,2)$, and $\left(2+(\dot{f})^{2}\right)^{-1 / 2}$, with $P_{i}$ and $\hat{P}_{0}$ vanishing identically on $S^{1}$ if $\left(f, \kappa_{1}, \kappa_{2}\right)=\left(0, \kappa_{0}, \kappa_{0}\right)$. Here $\hat{\phi}, k$, and $k_{i}^{\prime}$ are computed at $\left(q, \kappa_{1}, \kappa_{2}\right)$ rather than at $\left(p, \kappa_{1}, \kappa_{2}\right)$, and in passing from (6.13) to (6.18) we use the fact that $\theta_{q}, \hat{\phi}_{\left(q, \kappa_{1}, \kappa_{2}\right)}, k_{\left(q, \kappa_{1}, \kappa_{2}\right)}$, and $\left(k_{i}^{\prime}\right)_{\left(q, \kappa_{1}, \kappa_{2}\right)}$ are obtained by right-composing with $\sigma_{q(f)}$ the corresponding functions for $\left(p, \kappa_{1}, \kappa_{2}\right)$, as well as the fact that for any function $h,\left(h \circ \sigma_{q(f)}\right)^{\dot{ }}=\left(\dot{h} \circ \sigma_{q(f)}\right) \dot{\sigma}_{q(f)}=\left(\dot{h} \ell_{p}\left(2+\dot{f}^{2}\right)^{-1 / 2}\right) \circ \sigma_{q(f)}$.

Observe that $\tan \theta_{q}=\tan ^{-1}((1-\dot{f} / \sqrt{2}) /(1+\dot{f} / \sqrt{2}))$, so that $\theta_{q}-\pi / 4=$ $-\tan ^{-1}(\dot{f} / \sqrt{2})$. Hence in the list of variables in the preceding paragraph we may remove $\dot{\theta}_{q}$ and $\ddot{\theta}_{q}$ if we add $f^{(3)}$, and we also have the simplification

$$
\left.\left(2+\dot{f}^{2}\right)^{-1 / 2} f_{1} \dot{\theta}_{q}=-\sqrt{2}\left(2+\dot{f}^{2}\right)^{-3 / 2}\right) f_{1} .
$$

Similarly keeping track of the dependence of other variables in the list on $f, \kappa_{1}, \kappa_{2}$ and their derivatives, and using Corollary 6.3 to estimate $\left\|\left(2+\dot{f}^{2}\right)^{-1 / 2}\right\|_{n}$ and $\left\|\left(2+\dot{f}^{2}\right)^{-1}\right\|_{n}$, we see that on $W_{1}$ we have

$$
\left\|P_{i}\right\|_{n},\left\|\hat{P}_{0}\right\|_{n} \leq C\left\|\left(f, \kappa_{1}-\kappa_{0}, \kappa_{2}-\kappa_{0}\right)\right\|_{n+3}, \quad n \geq 0, \quad-1 \leq i \leq 2,
$$

for some constants $C=C\left(n, \kappa_{0}\right)$ that will be continually updated below and allowed to depend on $\varepsilon$ as well. Note that on $W_{\epsilon}$ we have

$$
\left\|P_{i}\right\|_{3} \leq C \epsilon, \quad\left\|\hat{P}_{0}\right\|_{3} \leq C \epsilon .
$$

Henceforth we assume $\left(f, \kappa_{1}, \kappa_{2}\right) \in W_{\epsilon}$, with $\epsilon$ to be determined later; every time we show that something is true for $\epsilon$ sufficiently small, from that point on we assume $\epsilon$ to be that small.

Now let $f_{1}=A_{0, \kappa_{0}}^{-1}\left(g_{1}\right)$. Since $A_{0, \kappa_{0}}$ is a positive-definite second-order elliptic operator on a compact manifold we have

$$
\left\|f_{1}\right\|_{L_{n}^{2}} \leq C\left\|g_{1}\right\|_{L_{\max }^{2}\{0, n-2\}}
$$

(see GT). From (6.18) we have

$$
\left\|A_{f, \kappa_{1}, \kappa_{2}} \circ A_{0, \kappa_{0}}^{-1}\left(g_{1}\right)-g_{1}\right\|_{L^{2}} \leq C \epsilon\left(\left\|f_{1}\right\|_{L_{2}^{2}}+\left|c\left(f ; f_{1}\right)\right|+\left\|\hat{\lambda}_{q}\left(\left(2+\dot{f}^{2}\right)^{-1 / 2} f_{1}\right)\right\|_{L_{2}^{2}}\right) .
$$

From (6.15), integrating by parts the term in (6.15) proportional to $\dot{f}_{1}$, we find

$$
\left|c\left(f ; f_{1}\right)\right| \leq C \epsilon\left\|f_{1}\right\|_{L^{2}} .
$$

We also have

$$
\left|\left\langle\left(2+\dot{f}^{2}\right)^{-3 / 2} \ddot{f} f_{1}\right\rangle\right| \leq C \epsilon\left\|f_{1}\right\|_{L^{2}},
$$


and since the $L^{2}$ norm of any function on $S^{1}$ with average value 0 is bounded by the $L^{2}$ norm of its derivative, using [G, equations (4.8) and (4.7)] and (6.19) we have

$$
\begin{aligned}
\left\|\hat{\lambda}_{q}\left(\left(2+\dot{f}^{2}\right)^{-1 / 2} f_{1}\right)\right\|_{L^{2}} \leq C\left\|\left(2+\dot{f}^{2}\right)^{-1 / 2} f_{1} \dot{\theta}_{q}\right\|_{L^{2}} & =C\left\|\left(2+\dot{f}^{2}\right)^{-3 / 2} \ddot{f} f_{1}\right\|_{L^{2}} \\
& \leq C \epsilon\left\|f_{1}\right\|_{L^{2}} .
\end{aligned}
$$

Using (6.22) with $n=0$, 6.23) therefore implies

$$
\left\|A_{f, \kappa_{1}, \kappa_{2}} \circ A_{0, \kappa_{0}}^{-1}-I\right\|_{\mathrm{op}, L^{2}} \leq C \epsilon,
$$

where the norm indicated is the operator norm on $\operatorname{Hom}\left(L^{2}\left(S^{1}\right)\right)$. It follows that for $\epsilon$ sufficiently small, $A_{f, \kappa_{1}, \kappa_{2}} \circ A_{0, \kappa_{0}}^{-1}$ extends to a topological isomorphism $L^{2}\left(S^{1}\right) \rightarrow$ $L^{2}\left(S^{1}\right)$.

Thus for $g_{2} \in C^{\infty}\left(S^{1}\right)$ we have $g_{1}:=\left(A_{f, \kappa_{1}, \kappa_{2}} \circ A_{0, \kappa_{0}}^{-1}\right)^{-1} g_{2} \in L^{2}$; we proceed to show that $g_{1} \in C^{\infty}$. As above, let $f_{1}=A_{0, \kappa_{0}}^{-1}\left(g_{1}\right)$. From (6.18) we have

$$
g_{1}=g_{2}-P_{2} \ddot{f}_{1}-R\left(f, \kappa_{1}, \kappa_{2} ; f_{1}\right),
$$

where $R\left(f, \kappa_{1}, \kappa_{2} ; f_{1}\right)$ consists of the right-hand side of (6.18) with the first two terms excluded. Since $A_{0, \kappa_{0}}\left(f_{1}\right)=g_{1}$, we can use (6.14) to solve for $\ddot{f}_{1}$ in terms of $g_{1}, \dot{f}_{1}$, and $f_{1}$; we then substitute the result into (6.27) and solve for $g_{1}$ in terms of the remaining objects. The result is

$$
\begin{aligned}
g_{1}=\frac{1}{1+P_{2} \xi_{2}} & \left\{g_{2}-\left(P_{1}+\xi_{1}\right) \dot{f}_{1}-\left(P_{0}+\xi_{0}\right) f_{1}\right. \\
& -P_{-1}\left\{\hat{\lambda}_{q}\left(\left(2+\dot{f}^{2}\right)^{-1 / 2} f_{1}\right)+c\left(f ; f_{1}\right)\right\} \\
& \left.-\hat{P}_{0}\left\langle-\sqrt{2}\left(2+\dot{f}^{2}\right)^{-3 / 2} \ddot{f} f_{1}\right\rangle\right\},
\end{aligned}
$$

where the $\xi_{i}$ are $C^{\infty}$ functions depending only on $\kappa_{0}$ (and $\varepsilon$ ) and where we have taken $\epsilon$ small enough to ensure that $1+P_{2} \xi_{2} \geq 1 / 2$ (see (6.21) $)$. Since $g_{1} \in L^{2}$ we have $f_{1} \in L_{2}^{2}$, so the right-hand side of (6.28) lies in $L_{1}^{2}$, and therefore $g_{1} \in L_{1}^{2}$. If $g_{1} \in L_{j}^{2}$, then the same argument shows $g_{1} \in L_{j+1}^{2}$. Hence $g_{1} \in L_{j}^{2}$ for all $j$, and therefore is $C^{\infty}$ by the Sobolev Embedding Theorem (see [A]).

We will use (6.28) to derive most of the remaining estimates we need, but to start the bootstrapping we return to (6.27). We have already seen that $\left|c\left(f ; f_{1}\right)\right| \leq$ $C \epsilon\left\|f_{1}\right\|_{L^{2}}$ and that $\left\|\hat{\lambda}_{q}\left(f_{1}\left(2+\dot{f}^{2}\right)^{-1 / 2}\right)\right\|_{L^{2}} \leq C \epsilon\left\|f_{1}\right\|_{L^{2}}$. Thus, using (6.27) and (6.22) we have

$$
\left\|g_{1}\right\|_{L^{2}} \leq C\left(\left\|g_{2}\right\|_{L^{2}}+\epsilon\left\|f_{1}\right\|_{L_{2}^{2}}\right) \leq C\left(\left\|g_{2}\right\|_{0}+\epsilon\left\|g_{1}\right\|_{L^{2}}\right) .
$$

Therefore for $\epsilon$ sufficiently small we have

$$
\left\|g_{1}\right\|_{L^{2}} \leq C\left\|g_{2}\right\|_{0} .
$$

Next, letting $h=1+P_{2} \xi_{2}$ and using (6.28), for $n \geq 1$ we have

$$
\begin{aligned}
\left\|g_{1}\right\|_{L_{n}^{2}} \leq & \left\|g_{2} h^{-1}\right\|_{L_{n}^{2}}+\left\|\left(P_{1}+\xi_{1}\right) h^{-1} \dot{f}_{1}\right\|_{L_{n}^{2}}+\left\|\left(P_{0}+\xi_{0}\right) h^{-1} f_{1}\right\|_{L_{n}^{2}} \\
& +\left\|P_{-1} h^{-1} \hat{\lambda}_{q}\left(2+\dot{f}^{2}\right)^{-1 / 2} f_{1}\right\|_{L_{n}^{2}}+\left\|P_{-1} h^{-1}\right\|_{n}\left|c\left(f ; f_{1}\right)\right| \\
& +\left\|\hat{P}_{0} h^{-1}\right\|_{n}\left|\left\langle\left(2+\dot{f}^{2}\right)^{-3 / 2} f_{1}\right\rangle\right| .
\end{aligned}
$$

For the first term in (6.30) we have

$$
\left\|g_{2} h^{-1}\right\|_{L_{n}^{2}} \leq\left\|g_{2} h^{-1}\right\|_{n} \leq C\left(\left\|h^{-1}\right\|_{n}\left\|g_{2}\right\|_{0}+\left\|h^{-1}\right\|_{0}\left\|g_{2}\right\|_{n}\right)
$$


using (6.3). Let $\zeta_{i}=\kappa_{i}-\kappa_{0}$ for $i=1,2$. Since $h \geq 1 / 2$, using Lemma 6.2 and (6.20) we have $\left\|h^{-1}\right\|_{n} \leq C\left(1+\left\|\left(f, \zeta_{1}, \zeta_{2}\right)\right\|_{n+3}\right)$. We then have

$$
\left\|g_{2} h^{-1}\right\|_{L_{n}^{2}} \leq C\left(1+\left\|\left(f, \zeta_{1}, \zeta_{2}\right)\right\|_{n+3}\left\|g_{2}\right\|_{0}+\left\|g_{2}\right\|_{n}\right) .
$$

For the second term in (6.30), first we use (6.3) and (6.20) to compute

$$
\left\|\left(P_{1}+\xi_{1}\right) h^{-1}\right\|_{n} \leq C\left(1+\left\|\left(f, \zeta_{1}, \zeta_{2}\right)\right\|_{n+3}\right) .
$$

Then, using Lemma 6.1, for $n=1$ we have

$$
\left\|\left(P_{1}+\xi_{1}\right) h^{-1} \dot{f}_{1}\right\|_{1} \leq C\left\|\left(P_{1}+\xi_{1}\right) h^{-1}\right\|_{1}\left\|\dot{f}_{1}\right\|_{L_{1}^{2}} \leq C\left(1+\left\|\left(f, \zeta_{1}, \zeta_{2}\right)\right\|_{4}\right)\left\|f_{1}\right\|_{L_{2}^{2}},
$$

while for $n \geq 2$ we have

$$
\begin{aligned}
\left\|\left(P_{1}+\xi_{1}\right) h^{-1} \dot{f}_{1}\right\|_{n} \leq & C\left(\left\|\left(P_{1}+\xi_{1}\right) h^{-1}\right\|_{n}\left\|\dot{f}_{1}\right\|_{L_{2}^{2}}+\left\|\left(P_{1}+\xi_{1}\right) h^{-1}\right\|_{1}\left\|\dot{f}_{1}\right\|_{L_{n}^{2}}\right) \\
\leq & C\left(\left(1+\left\|\left(f, \zeta_{1}, \zeta_{2}\right)\right\|_{n+3}\right)\left\|f_{1}\right\|_{L_{3}^{2}}\right. \\
& \left.+\left(1+\left\|\left(f, \zeta_{1}, \zeta_{2}\right)\right\|_{4}\right)\left\|f_{1}\right\|_{L_{n+1}^{2}}\right) .
\end{aligned}
$$

The bound on the third term in (6.30) is identical except that the order of the derivative of $f_{1}$ is reduced by 1 ; hence this bound is dominated by the bound on the second term. For the last three terms in (6.30), let $P$ denote $P_{-1}$ or $\hat{P}_{0}$; from (6.3) and (6.20) we have $\|P\|_{n} \leq C\left(1+\epsilon\left\|\left(f, \zeta_{1}, \zeta_{2}\right)\right\|_{n+3}\right)$. Also, since $\left\langle\hat{\lambda}_{q}(g)\right\rangle=0$ for any $g$, we have $\left\|\hat{\lambda}_{q}(g)\right\|_{L_{n}^{2}} \leq C(n)\left\|\hat{\lambda}_{q}(g)\right\|_{L_{\max \{n-1,0\}}^{2}}$, and hence for $n \geq 1$

$$
\left\|\hat{\lambda}_{q}\left(\left(2+\dot{f}^{2}\right)^{-1 / 2} f_{1}\right)\right\|_{L_{n}^{2}} \leq\left\|\left(2+\dot{f}^{2}\right)^{-1 / 2} f_{1} \dot{\theta}_{q}\right\|_{L_{n-1}^{2}}=C\left\|\ddot{f}\left(2+\dot{f}^{2}\right)^{-3 / 2} f_{1}\right\|_{L_{n-1}^{2}}
$$

(cf. (6.26) $)$. Thus we have $\left\|\hat{\lambda}_{q}\left(\left(2+\dot{f}^{2}\right)^{-1 / 2} f_{1}\right)\right\|_{L_{1}^{2}} \leq C\|f\|_{2}\left\|f_{1}\right\|_{L_{1}^{2}} \leq C \epsilon\left\|f_{1}\right\|_{L^{2}}$, and similarly $\left\|\hat{\lambda}_{q}\left(\left(2+\dot{f}^{2}\right)^{-1 / 2} f_{1}\right)\right\|_{L_{2}^{2}} \leq C \epsilon\left\|f_{1}\right\|_{L_{1}^{2}}$. For $n \geq 3$, (6.32) and Lemma 6.1, in conjunction with (6.3) and Lemma 6.2, yield

$$
\begin{aligned}
\left\|\hat{\lambda}_{q}\left(\left(2+\dot{f}^{2}\right)^{-1 / 2} f_{1}\right)\right\|_{L_{n}^{2}} \leq & C\left(\left\|\ddot{f}\left(2+\dot{f}^{2}\right)^{-3 / 2}\right\|_{n-1}\left\|f_{1}\right\|_{L_{2}^{2}}\right. \\
& \left.+\left\|\ddot{f}\left(2+\dot{f}^{2}\right)^{-3 / 2}\right\|_{1}\left\|f_{1}\right\|_{L_{n-1}^{2}}\right) \\
\leq & C\left(\|f\|_{n+1}\left\|f_{1}\right\|_{L_{2}^{2}}+\epsilon\left\|f_{1}\right\|_{L_{n-1}^{2}}\right) .
\end{aligned}
$$

Using these estimates, another application of Lemma 6.1 then yields

$$
\begin{aligned}
\left\|P_{-1} h^{-1} \hat{\lambda}_{q}\left(f_{1}\left(2+\dot{f}^{2}\right)^{-1 / 2}\right)\right\|_{L_{n}^{2} \leq} \leq \epsilon\{1 & \left.+\left\|\left(f, \zeta_{1}, \zeta_{2}\right)\right\|_{n+3}\right)\left\|f_{1}\right\|_{L^{2}} \\
& +\max \{0, n-2\}\left(\|f\|_{n+1}\|\|_{1} \|_{L_{2}^{2}}\right. \\
& \left.\left.+\epsilon\left\|f_{1}\right\|_{L_{n-1}^{2}}\right)\right\},
\end{aligned}
$$

which also is dominated by the bound (6.31) on the second term of (6.30). Using (6.20), (6.24), and (6.25), the fifth and sixth terms in (6.30) are bounded by $C \epsilon\left(1+\left\|\left(f, \zeta_{1}, \zeta_{2}\right)\right\|_{n+3}\right)$, which again is dominated by the bound on the second term.

Combining the bounds on the various terms of (6.30) and then using (6.22), we finally arrive at the bootstrap bound

$$
\begin{aligned}
\left\|g_{1}\right\|_{L_{n}^{2}} \leq C\{ & \left\|g_{2}\right\|_{n}+\left(1+\left\|\left(f, \zeta_{1}, \zeta_{2}\right)\right\|_{4}\right)\left\|g_{1}\right\|_{L_{n-1}^{2}} \\
& \left.\left(1+\left\|\left(f, \zeta_{1}, \zeta_{2}\right)\right\|_{n+3}\right)\left(1+\left\|g_{2}\right\|_{0}+\left\|g_{1}\right\|_{L_{\min \{1, n-1\}}^{2}}\right)\right\} .
\end{aligned}
$$

We now shrink the neighborhoods on which we have been working. Define the open set

$$
W_{\epsilon}^{\prime}=\left\{\left(f, \kappa_{1}, \kappa_{2}\right) \in W_{\epsilon} \mid\left\|\left(f, \kappa_{1}-\kappa_{0}, \kappa_{2}-\kappa_{0}\right)\right\|_{4}<1\right\} \subset U \times V \times V,
$$


and let $B$ be an open ball of unit $C^{1}$-radius in $C^{\infty}\left(S^{1}\right)$ centered at some point $g_{0}$. Henceforth assume that $\left(f, \kappa_{1}, \kappa_{2}\right) \in W_{\epsilon}^{\prime}$ and that $g_{2} \in B$. Then for $n=1$, (6.33) and (6.29) imply $\left\|g_{1}\right\|_{L_{1}^{2}} \leq C$. For $n \geq 2$ the terms $\left\|\left(f, \zeta_{1}, \zeta_{2}\right)\right\|_{4},\left\|g_{2}\right\|_{0}$ and $\left\|g_{1}\right\|_{L_{\min \{1, n-1\}}^{2}}\left(=\left\|g_{1}\right\|_{L_{1}^{2}}\right)$ are now uniformly bounded, so we have simply

$$
\left\|g_{1}\right\|_{L_{n}^{2}} \leq C\left(1+\left\|g_{2}\right\|_{n}+\left\|\left(f, \zeta_{1}, \zeta_{2}\right)\right\|_{n+3}+\left\|g_{1}\right\|_{L_{n-1}^{2}}\right) .
$$

It then easily follows by induction that $\left\|g_{1}\right\|_{L_{n}^{2}} \leq C\left(1+\left\|g_{2}\right\|_{n}+\left\|\left(f, \zeta_{1}, \zeta_{2}\right)\right\|_{n+3}\right)$. Finally, using the Sobolev inequality (6.1), we arrive at

$$
\left\|\left(A_{f, \kappa_{1}, \kappa_{2}} \circ A_{0, \kappa_{0}}^{-1}\right)^{-1} g_{2}\right\|_{n}=\left\|g_{1}\right\|_{n} \leq C\left(1+\left\|g_{2}\right\|_{n+1}+\left\|\left(f, \zeta_{1}, \zeta_{2}\right)\right\|_{n+4}\right) .
$$

Hence the map $\left(f, \kappa_{1}, \kappa_{2}, g_{2}\right) \mapsto\left(A_{f, \kappa_{1}, \kappa_{2}} \circ A_{0, \kappa_{0}}^{-1}\right)^{-1} g_{2}$ is tame, and therefore so is the map $\left(f, \kappa_{1}, \kappa_{2}, f_{1}\right) \mapsto\left(A_{f, \kappa_{1}, \kappa_{2}}\right)^{-1}\left(f_{1}\right)$.

For all $f$ lying in the projection onto the first factor of $W_{\epsilon}^{\prime}$, define $S_{f}: C^{\infty}\left(S^{1}\right) \rightarrow$ $C^{\infty}\left(S^{1}\right)$ by $S_{f}(g)=g \circ \sigma_{q(f)}$; then $S_{f}$ is invertible with $S_{f}^{-1}(g)=g \circ \sigma_{q(f)}^{-1}$. Hence, from (6.16), $\left.D^{(1)} G^{\varepsilon}\right|_{\left(f, \kappa_{1}, \kappa_{2}\right)}=S_{f} \circ A_{f, \kappa_{1}, \kappa_{2}}$ is invertible, with

$$
\left(\left.D^{(1)} G^{\varepsilon}\right|_{\left(f, \kappa_{1}, \kappa_{2}\right)}\right)^{-1}\left(f_{1}\right)=\left(A_{f, \kappa_{1}, \kappa_{2}}\right)^{-1} \circ S_{f}^{-1}\left(f_{1}\right) .
$$

The map $f \mapsto \sigma_{q(f)}^{-1}$ is just the restriction to $\{a=0\}$ of the tame map $G_{5} \circ G_{1}$ in the proof of [G, Theorem 2.8], hence is tame, and the map $G_{8}: C^{\infty}\left(S^{1}\right) \times \operatorname{Diff}^{+}\left(S^{1}\right)$ defined by $G_{9}(g, \sigma)=g \circ \sigma$, is also tame; therefore so is the map $G_{10}:\left(f, f_{1}\right) \mapsto$ $S_{f}^{-1}\left(f_{1}\right)$. Since tameness is preserved under composition, we conclude that the map

$$
\begin{aligned}
K: W_{\epsilon}^{\prime} \times B & \rightarrow C^{\infty}\left(S^{1}\right), \\
\left(f, \kappa_{1}, \kappa_{2}, f_{1}\right) & \mapsto\left(\left.D^{(1)} G^{\varepsilon}\right|_{f, \kappa_{1}, \kappa_{2}}\right)^{-1}\left(f_{1}\right),
\end{aligned}
$$

is tame.

Just as in the proof of [G. Theorem 2.8], to satisfy the hypotheses of the Tame Implicit Function Theorem it remains only to show that $K$ is continuous. It is easily seen that the map $G_{10}$ is continuous and that the fixed linear map $A_{0, \kappa_{0}}$ is continuous, so it suffices to show that the map $G_{11}:\left(f, \kappa_{1}, \kappa_{2}, g\right) \mapsto\left(A_{f, \kappa_{1}, \kappa_{2}} \circ\right.$ $\left.A_{0, \kappa_{0}}^{-1}\right)^{-1} g$ is continuous.

From (6.18) it is not hard to check that for each $n$ there is a constant $C(n)$ such that for all $\left(f, \kappa_{1}, \kappa_{2}\right),\left(f^{\prime}, \kappa_{1}^{\prime}, \kappa_{2}^{\prime}\right) \in W_{\epsilon}^{\prime}$,

$$
\left\|\left(A_{f^{\prime}, \kappa_{1}^{\prime}, \kappa_{2}^{\prime}} \circ A_{0, \kappa_{0}}^{-1}\right)\left(f_{1}\right)-\left(A_{f, \kappa_{1}, \kappa_{2}} \circ A_{0, \kappa_{0}}^{-1}\right)\left(f_{1}\right)\right\|_{L_{n}^{2}} \leq C(n)\left\|\left(\begin{array}{c}
f^{\prime} \\
\kappa_{1}^{\prime} \\
\kappa_{2}^{\prime}
\end{array}\right)-\left(\begin{array}{c}
f \\
\kappa_{1} \\
\kappa_{2}
\end{array}\right)\right\|_{L_{n}^{2}},
$$

and thus the map

$$
\begin{aligned}
W_{\epsilon}^{\prime} & \rightarrow \operatorname{Hom}\left(C^{\infty}\left(S^{1}\right)\right), \\
\left(f, \kappa_{1}, \kappa_{2}\right) & \mapsto A_{f, \kappa_{1}, \kappa_{2}} \circ A_{0, \kappa_{0}}^{-1}
\end{aligned}
$$

is continuous with respect to the $(n+4)$-norm topology on $W_{\epsilon}^{\prime}$ and the (op, $L_{n+1}^{2}$ )norm topology on $\operatorname{Hom}\left(C^{\infty}\left(S^{1}\right)\right)$. Since inversion is a continuous map

$$
\operatorname{Aut}\left(L_{n+1}^{2}\left(S^{1}\right)\right) \rightarrow \operatorname{Aut}\left(L_{n+1}^{2}\left(S^{1}\right)\right),
$$

it follows that the map $\left(f, \kappa_{1}, \kappa_{2}\right) \mapsto A_{f, \kappa_{1}, \kappa_{2}} \circ A_{0, \kappa_{0}}^{-1}$ is also continuous with respect to the stated topologies on source and target. This continuity in the operator-norm topology then implies that the map $G_{11}$ is continuous as a map 
$\left(W_{\epsilon}^{\prime},\|\|_{n+4}\right) \times\left(B,\|\|_{L_{n+1}^{2}}\right) \rightarrow\left(C^{\infty}\left(S^{1}\right),\|\|_{L_{n+1}^{2}}\right)$, and hence, using the obvious embedding $C^{n+1}\left(S^{1}\right) \rightarrow L_{n+1}^{2}\left(S^{1}\right)$ and the Sobolev embedding $L_{n+1}^{2}\left(S^{1}\right) \rightarrow C^{n}\left(S^{1}\right)$, it follows that $G_{11}$ is continuous as a map $\left(W_{\epsilon}^{\prime},\|\|_{n+4}\right) \times\left(B,\|\|_{n+1}\right) \rightarrow\left(C^{\infty}\left(S^{1}\right),\|\|_{n}\right)$. Since this is true for all $n, G_{11}$ is continuous with respect to the $C^{\infty}$ topologies on $W_{\epsilon}^{\prime} \times B$ and $C^{\infty}\left(S^{1}\right)$.

The Tame Implicit Function Theorem now implies that there exist $C^{\infty}$-open neighborhoods $U_{0}$ of $\left(\kappa_{0}, \kappa_{0}\right)$ in $C^{\infty}\left(S^{1}\right) \times C^{\infty}\left(S^{1}\right)$ and $W$ of 0 in $C^{\infty}\left(S^{1}\right)$, such that for all $\left(\kappa_{1}, \kappa_{2}\right) \in U_{0}$, the equation $G^{\varepsilon}\left(f, \kappa_{1}, \kappa_{2}\right)=0$ has a unique solution $f$ in $W$; equivalently, an open neighborhood $\bar{W}$ of $\Delta$ in $\mathcal{M}^{\text {int }}$ on which the equation $Z_{\kappa_{1}, \kappa_{2}}^{\varepsilon}(\Sigma)=0$ has a unique solution $\Sigma$; equivalently, an open neighborhood $\tilde{W}$ of $p^{\Delta}$ in $\widetilde{\mathcal{M}}^{\text {int,c }}$ for which the equation $\tilde{Z}_{\kappa_{1}, \kappa_{2}}^{\varepsilon}(p)=0$ has a unique solution $p$ in $W^{\prime}:=\tilde{W} \cap \mathcal{H}^{c}$. Let us denote these solutions of $Z_{\kappa_{1}, \kappa_{2}}^{\varepsilon}(\cdot)=0$ and $\tilde{Z}_{\kappa_{1}, \kappa_{2}}^{\varepsilon}(p)=0$ by $\Sigma\left(\kappa_{1}, \kappa_{2}\right)$ and $p\left(\kappa_{1}, \kappa_{2}\right)$ respectively.

By definition of the $C^{\infty}$ topology, $U_{0}$ contains for some $j_{1}$ an open ball of finite $C^{j_{1}}$-radius $r_{1}$, so without loss of generality we may take $U_{0}$ to be such a ball. Our estimates on $D^{(1)} G^{\varepsilon}$ then imply that $W$ is also contained in a ball of finite $C^{j_{2}}$. radius $r_{2}$ for some $j_{2}$. By the Tame Implicit Function Theorem the map Crit : $U_{0} \rightarrow W,\left(\kappa_{1}, \kappa_{2}\right) \mapsto \Sigma\left(\kappa_{1}, \kappa_{2}\right)$ is continuous (in fact, tamely smooth), as is the corresponding map Crit $^{\prime}:\left(\kappa_{1}, \kappa_{2}\right) \mapsto p\left(\kappa_{1}, \kappa_{2}\right) \in W^{\prime}$, so we may shrink $W$ or $W^{\prime}$ to any desired open set, replacing $U_{0}$ by the new inverse image $\operatorname{Crit}^{-1}\left(W^{\prime}\right)$ or $\left(\text { Crit }^{\prime}\right)^{-1}\left(W^{\prime}\right)$. In particular by increasing $j_{1}$ if necessary we may assume $j_{2} \geq 4$, and by decreasing $r_{1}$ if necessary we may assume that there is some $\delta>0$ such that for all $p=\left(p_{1}, p_{2}\right) \in W^{\prime}$ we have $\dot{p}_{i}(t) \geq \delta$ for all $t \in S^{1}, i=1,2$. In particular, (the new) $W^{\prime}$ lies in the interior of $\widetilde{\mathcal{B}}^{\text {int }, c}$. We shrink $W$ further, if necessary, to ensure that for all $f \in W$ we have $\|f\|_{1} \leq \epsilon_{3}$, where $\epsilon_{3} \leq 1$ will be determined below.

Fix $\left(\kappa_{1}, \kappa_{2}\right) \in U_{0}$. So far we know only that $\Sigma\left(\kappa_{1}, \kappa_{2}\right)$ (resp. $\left.p\left(\kappa_{1}, \kappa_{2}\right)\right)$ is the unique critical point of $J_{\left(\kappa_{1}, \kappa_{2}\right)}^{\varepsilon}\left(\operatorname{resp} . \tilde{J}_{\left(\kappa_{1}, \kappa_{2}\right)}^{c, \varepsilon}\right)$ in $W\left(\operatorname{resp} . W^{\prime}\right)$. We still must show that, shrinking $U_{0}$ (and correspondingly $W$ and $W^{\prime}$ ) if necessary, $\left.J_{\left(\kappa_{1}, \kappa_{2}\right)}^{\varepsilon}\right|_{W}$ achieves an absolute minimum at $\Sigma\left(\kappa_{1}, \kappa_{2}\right)$ and nowhere else (equivalently, that $p\left(\kappa_{1}, \kappa_{2}\right)$ uniquely minimizes $\tilde{J}_{\left(\kappa_{1}, \kappa_{2}\right)}^{c, \varepsilon}$ globally on $\left.W^{\prime}\right)$.

Let $p_{1}=p\left(\kappa_{1}, \kappa_{2}\right)$, let $f_{1}=\left(\Psi_{p^{\Delta}}^{c}\right)^{-1}\left(p_{1}\right)$, let $f_{2} \in W$, and let $p_{2}=\Psi_{p^{\Delta}}^{c}\left(f_{2}\right)$. Since $\tilde{J}_{\left(\kappa_{1}, \kappa_{2}\right)}^{c, \varepsilon}$ is $\operatorname{Diff}^{+}\left(S^{1}\right)$-invariant, we have

$$
\begin{aligned}
\tilde{J}_{\left(\kappa_{1}, \kappa_{2}\right)}^{c, \varepsilon}\left(p_{2}\right)=\tilde{J}_{\left(\kappa_{1}, \kappa_{2}\right)}^{c, \varepsilon}\left(q\left(f_{2}\right) \circ \sigma_{q\left(f_{2}\right)}\right) & =\tilde{J}_{\left(\kappa_{1}, \kappa_{2}\right)}^{c, \varepsilon}\left(q\left(f_{2}\right)\right) \\
& =\tilde{J}_{\left(\kappa_{1}, \kappa_{2}\right)}^{c, \varepsilon}\left(q\left(f_{1}\right)+\left(f_{2}-f_{1}\right) \mathbf{N}_{p^{\Delta}}\right) \\
& =\tilde{J}_{\left(\kappa_{1}, \kappa_{2}\right)}^{c, \varepsilon}\left(\left(q\left(f_{1}\right)+\left(f_{2}-f_{1}\right) \mathbf{N}_{p^{\Delta}}\right) \circ \sigma_{q\left(f_{1}\right)}\right) \\
& =\tilde{J}_{\left(\kappa_{1}, \kappa_{2}\right)}^{c, \varepsilon}\left(p_{1}+X\right)
\end{aligned}
$$

where $X=\left[\left(f_{2}-f_{1}\right) \mathbf{N}_{p^{\Delta}}\right] \circ \sigma_{q\left(f_{1}\right)}$.

Let $Y=\left(f_{2}-f_{1}\right) \mathbf{N}_{p^{\Delta}}$. From Lemma 6.1 we have $\|Y\|_{L_{1}^{2}} \leq C\left\|f_{2}-f_{1}\right\|_{L_{1}^{2}}$. By a change-of-variables in the integrals defining $\left\|Y \circ \sigma_{q\left(f_{1}\right)}\right\|_{L_{1}^{2}}^{2}$, using the fact that $\left(\sigma_{q\left(f_{1}\right)}^{-1}\right)^{\cdot}(s)=\ell_{q}^{-1}\left(2+\dot{f}_{1}^{2}(s)\right)^{1 / 2}$, we see that $\left|\left\|Y \circ \sigma_{q\left(f_{1}\right)}\right\|_{L_{1}^{2}}-\|Y\|_{L_{1}^{2}}\right| \leq C \epsilon_{3}\|Y\|_{L_{1}^{2}}$, so $\|X\|_{L_{1}^{2}} \leq C\left\|f_{2}-f_{1}\right\|_{L_{1}^{2}}$. Since $p_{1}$ is a critical point of $\tilde{J}_{\left(\kappa_{1}, \kappa_{2}\right)}^{\varepsilon}$, Lemma 6.4 implies 
that for all $\epsilon_{1}>0$ there exists $\epsilon^{\prime}=\epsilon^{\prime}\left(\epsilon_{1}\right)>0$ such that if $\left\|f_{2}-f_{1}\right\|_{L_{1}^{2}}<\epsilon^{\prime}$, then

$$
\tilde{J}_{\left(\kappa_{1}, \kappa_{2}\right)}^{\varepsilon}\left(p_{1}+X\right) \geq \tilde{J}_{\left(\kappa_{1}, \kappa_{2}\right)}^{\varepsilon}\left(p_{1}\right)+\left.\frac{1}{2} H \tilde{J}_{\left(\kappa_{1}, \kappa_{2}\right)}^{\varepsilon}\right|_{p_{1}}(X, X)-C \epsilon_{1}\left\|f_{2}-f_{1}\right\|_{L_{1}^{2}}^{2} .
$$

From the definition of $q\left(f_{1}\right)$ we have $\mathbf{T}_{q\left(f_{1}\right)}=\left(2+\dot{f}_{1}^{2}\right)^{-1 / 2}\left(\sqrt{2} \mathbf{T}_{p^{\Delta}}+\dot{f}_{1} \mathbf{N}_{p^{\Delta}}\right)$, implying $\mathbf{N}_{q\left(f_{1}\right)}=\left(2+\dot{f}_{1}^{2}\right)^{-1 / 2}\left(\sqrt{2} \mathbf{N}_{p^{\Delta}}-\dot{f}_{1} \mathbf{T}_{p^{\Delta}}\right)$. Inverting this relationship we find $\mathbf{N}_{p^{\Delta}}=\left(2+\dot{f}_{1}^{2}\right)^{-1 / 2}\left(\sqrt{2} \mathbf{N}_{q\left(f_{1}\right)}+\dot{f}_{1} \mathbf{T}_{q\left(f_{1}\right)}\right)$. Since $\mathbf{N}_{q\left(f_{1}\right)} \circ \sigma_{q\left(f_{1}\right)}=\mathbf{N}_{p_{1}}$ and $\mathbf{T}_{q\left(f_{1}\right)} \circ \sigma_{q\left(f_{1}\right)}=\mathbf{T}_{p_{1}}$, we therefore have

$$
X=\left[\left(f_{2}-f_{1}\right) \circ \sigma_{q\left(f_{1}\right)}\right]\left(2+\dot{f}_{1}^{2}\right)^{-1 / 2}\left(\sqrt{2} \mathbf{N}_{p_{1}}+\dot{f}_{1} \mathbf{T}_{p_{1}}\right) .
$$

Let $\epsilon_{2}:=\epsilon_{2}\left(\kappa_{0}, \varepsilon\right)$ be as in Lemma 6.5, and shrink $W$ further if necessary to ensure that $\left\|\Psi_{p^{\Delta}}^{c}(f)-p^{\Delta}\right\|_{3}<\epsilon_{2} \|$ for all $f \in W$. Then the lemma implies that the Hessian term in (6.34) depends only on the $p_{1}$-normal component of $X$ and that

$$
\begin{aligned}
\left.H \tilde{J}_{\left(\kappa_{1}, \kappa_{2}\right)}^{\varepsilon}\right|_{p}(X, X) & \geq c_{1}\left(\kappa_{0}\right)\left\|\left[\left(f_{2}-f_{1}\right) \circ \sigma_{q\left(f_{1}\right)}\right]\left(2+\dot{f}_{1}^{2}\right)^{-1 / 2}\right\|_{L_{1}^{2}}^{2} \\
& \geq c_{1}\left(\kappa_{0}\right)\left\|f_{2}-f_{1}\right\|_{L_{1}^{2}}^{2}\left(1-C \epsilon_{3}\right),
\end{aligned}
$$

where the last inequality follows from the same argument applied to $\left\|Y \circ \sigma_{q\left(f_{1}\right)}\right\|_{L_{1}^{2}}$ above.

We now assume that $\epsilon_{3}$ has been chosen so that for the value of $C$ in 6.35) we have $C \epsilon_{3}<\frac{1}{2}$. Then choose $\epsilon_{1}$ so that, for the value of $C$ in (6.34), we have $C \epsilon_{1} \leq \frac{1}{8} c_{1}\left(\kappa_{0}, \varepsilon\right)$. We then shrink $W$ further, if necessary, to ensure that its $C^{1}$ radius is less than $\epsilon^{\prime}\left(\epsilon_{1}\right) / 2$, so that $\left\|f_{2}-f_{1}\right\|_{L_{1}^{2}} \leq\left\|f_{2}-f_{1}\right\|_{1} \leq \epsilon^{\prime}\left(\epsilon_{1}\right)$. Then for $\left(\kappa_{1}, \kappa_{2}\right) \in \operatorname{Crit}^{-1}(W)$ and all $f_{2} \in W$ we have

$$
\tilde{J}_{\left(\kappa_{1}, \kappa_{2}\right)}^{\varepsilon}\left(\Psi_{p^{\Delta}}\left(f_{2}\right)\right) \geq \tilde{J}_{\left(\kappa_{1}, \kappa_{2}\right)}^{\varepsilon}\left(p\left(\kappa_{1}, \kappa_{2}\right)\right)+\frac{1}{8} c_{1}\left(\kappa_{0}, \varepsilon\right)\left\|f_{2}-\operatorname{Crit}\left(\kappa_{1}, \kappa_{2}\right)\right\|_{L_{1}^{2}}^{2} .
$$

Thus $p\left(\kappa_{1}, \kappa_{2}\right)$ is the unique global minimizer of $\left.\tilde{J}_{\left(\kappa_{1}, \kappa_{2}\right)}^{\varepsilon}\right|_{W^{\prime}}$.

\section{REFERENCES}

[A] R. Adams, Sobolev Spaces, Academic Press, New York, 1975. MR0450957 (56:9247)

[FB] M. Frenkel and R. Basri, Curve matching using the fast marching method, Energy Minimization Methods in Computer Vision and Pattern Recognition: Proc. 4th International Workshop, EMMCVPR 2003, A. Rangarajan et al. (eds.), Springer-Verlag, Berlin, 2003, pp. $35-51$.

[GT] D. Gilbarg and N. S. Trudinger, Elliptic Partial Differential Equations of Second Order, second edition. Springer-Verlag, Berlin, 1983. MR737190 (86c:35035)

[G] D. Groisser, Certain optimal correspondences between plane curves, I: manifolds of shapes and bimorphisms, Trans. Amer. Math. Soc., this issue.

[H] R. S. Hamilton, The Inverse Function Theorem of Nash and Moser, Bull. (New Ser.) Amer. Math. Soc. 7 (1982), 65-222. MR656198 (83j:58014)

[T] H. D. Tagare, Shape-based nonrigid correspondence with application to heart motion analysis, IEEE Trans. Med. Imaging 18 (1999), 570-579.

[TOG] H. D. Tagare, D. O'Shea, and D. Groisser, Non-rigid shape comparison of plane curves in images, J. Math. Imaging and Vision 16 (2002), 57-68. MR1884465 (2002m:68120)

Department of Mathematics, University of Florida, Gainesville, Florida 32611-8105

E-mail address: groisser@math.ufl.edu 